\title{
Structure and stability of recombinant bovine odorant-binding protein: I. Design and analysis of monomeric mutants
}

Olga V Stepanenko, Denis O Roginskii, Olesya V Stepanenko, Irina M Kuznetsova, Vladimir N Uversky, Konstantin K Turoverov

Bovine odorant-binding protein (bOBP) differs from other lipocalins by lacking the conserved disulfide bond and being able to form the domain-swapped dimers. To identify structural features responsible for the formation of the bOBP unique dimeric structure and to understand the role of the domain swapping on maintaining the native structure of the protein, structural properties of the recombinant wild type bOBP and its mutant that cannot dimerize via the domain swapping were analyzed. We also looked at the effect of the disulfide bond by designing a monomeric bOBPs with restored disulfide bond which is conserved in other lipocalins. Finally, to understand which features in the microenvironment of the bOBP tryptophan residues play a role in the defining peculiarities of the intrinsic fluorescence of this protein we designed and investigated single-tryptophan mutants of the monomeric bOBP. Our analysis revealed that the insertion of the glycine after the residue 121 of the bOBP prevents domain swapping and generates a stable monomeric protein bOBP-Gly121+. We also show that the restored disulfide bond in the GCC-bOBP mutant leads to the noticeable stabilization of the monomeric structure. Structural and functional analysis revealed that none of the amino acid substitutions introduced to the bOBP affected functional activity of the protein and that the ligand binding leads to the formation of a more compact and stable state of the recombinant bOBP and its mutant monomeric forms. Finally, analysis of the single-tryptophan mutants of the monomeric bOBP gave us a unique possibility to find peculiarities of the microenvironment of tryptophan residues which were not previously described. 


\section{Structure and stability of recombinant bovine odorant-binding}

\section{2 protein: I. Design and analysis of monomeric mutants}

4 Olga V. Stepanenko, ${ }^{1}$ Denis O. Roginskii, ${ }^{2}$ Olesya V. Stepanenko, ${ }^{1}$ Irina M. Kuznetsova, ${ }^{1,2}$

5 Vladimir N. Uversky,,$^{1,3, *}$ and Konstantin K. Turoverov ${ }^{1,2, *}$

$7{ }^{1}$ Laboratory of structural dynamics, stability and folding of proteins, Institute of Cytology,

8 Russian Academy of Sciences, St. Petersburg, Russia;

$9 \quad{ }^{2}$ Peter the Great St. Petersburg Polytechnic University, St. Petersburg, Russia;

10 32Department of Molecular Medicine and USF Health Byrd Alzheimer's Research Institute,

11 Morsani College of Medicine, University of South Florida, Tampa, FL, USA

12

*To whom correspondence should be addressed: VNU, Department of Molecular Medicine,

14 University of South Florida, 12901 Bruce B. Downs Blvd. MDC07, Tampa, Florida 33612, USA, E-mail: vuversky@health.usf.edu; KKT, Institute of Cytology, Russian Academy of Sciences, Tikhoretsky Av., 4, St. Petersburg 194064, Russia, E-mail: kkt@incras.ru

19 Running title: Monomeric forms of bOBP 


\section{ABSTRACT}

22 Bovine odorant-binding protein (bOBP) differs from other lipocalins by lacking the conserved

23 disulfide bond and being able to form the domain-swapped dimers. To identify structural features

24 responsible for the formation of the bOBP unique dimeric structure and to understand the role of

25 the domain swapping on maintaining the native structure of the protein, structural properties of the recombinant wild type bOBP and its mutant that cannot dimerize via the domain swapping were analyzed. We also looked at the effect of the disulfide bond by designing a monomeric bOBPs with restored disulfide bond which is conserved in other lipocalins. Finally, to understand which features in the microenvironment of the bOBP tryptophan residues play a role in the defining peculiarities of the intrinsic fluorescence of this protein we designed and investigated singletryptophan mutants of the monomeric bOBP. Our analysis revealed that the insertion of the glycine after the residue 121 of the bOBP prevents domain swapping and generates a stable monomeric protein bOBP-Gly121+. We also show that the restored disulfide bond in the GCC-bOBP mutant leads to the noticeable stabilization of the monomeric structure. Structural and functional analysis revealed that none of the amino acid substitutions introduced to the bOBP affected functional activity of the protein and that the ligand binding leads to the formation of a more compact and stable state of the recombinant bOBP and its mutant monomeric forms. Finally, analysis of the single-tryptophan mutants of the monomeric bOBP gave us a unique possibility to find peculiarities of the microenvironment of tryptophan residues which were not previously described. 
42 Abbreviations: bOBP, bovine odorant-binding protein; $\mathrm{pOBP}$, porcine odorant-binding protein;

$43 \mathrm{GdnHCl}$, guanidine hydrochloride; $\mathrm{CD}$, circular dichroism; UV, ultra-violet; Parameter $A$,

$44 \quad\left(\mathrm{I}_{320} / \mathrm{I}_{365}\right)$ upon excitation at $\lambda_{\mathrm{ex}}=297 \mathrm{~nm}$. 


\section{INTRODUCTION}

47

Lipocalins constitute a family of carrier proteins that transport various small hydrophobic molecules ranging from lipids to retinoids, steroids, and bilins. Being found in animals, plants, and bacteria and possessing low sequence identity (less than $20 \%$ ), these proteins are characterized by the presence of the conserved "lipocalin fold" that includes two structural modules, an eightstranded $\beta$-barrel that constitutes $70-80 \%$ of the protein and includes the ligand-binding site and a C-terminal $\alpha$-helix with unknown function (Flower et al. 2000). Evolution of the lipocalin fold generated numerous specialized carrier proteins with the highly diversified binding specificities. One of the sub-classes of the lipocalin family includes odorant binding proteins (OBPs) with the characteristic ability of reversible binding of various odorant molecules; i.e. volatile, small and hydrophobic compounds with no fixed structure and chemical properties (Tegoni et al. 2000). Classic odorant binding protein (OBP) is characterized by a specific monomeric fold, where the eight $\beta$-strands, a short $\alpha$-helical region, and the ninth $\beta$-strand interact to form a $\beta$-barrel followed by the disordered C-terminal tail (Bianchet et al. 1996; Flower et al. 2000). The ligand binding site of these proteins is formed by hydrophobic and aromatic residues located within the inner cavity of the $\beta$-barrel and loop regions connecting $\beta$-strands of the barrel. The conserved disulfide bridge formed by Cys 63 and Cys 155 is commonly found in many OBPs to link the flexible C-terminal moiety and strand $\beta 4$.

64 Curiously, despite rather high sequence identity between porcine and bovine OBPs (42\%), these lipocalins are characterized by different quaternary structures, with porcine OBP (pOBP) being a monomeric protein (Spinelli et al. 1998), and with bovine OBP (bOBP) being a dimer (Bianchet et al. 1996; Tegoni et al. 1996), protomers of which lack the disulfide bridge which is a common feature for all lipocalin family members (Tegoni et al. 2000). Therefore, bOBP has a 
69 unique dimeric structure, which is quite different from the monomeric folds of the majority of 70 classical OBPs (Bianchet et al. 1996; Stepanenko et al. 2014b) (Figure 1). In the bOBP dimer, each

71 of the two protomers forms a $\beta$-barrel that interacts with the $\alpha$-helical portion of the $\mathrm{C}$-terminal tail

72 of other protomer via the domain swapping mechanism. Such a mechanism was described for

73 many dimeric and oligomeric protein complexes, where it plays important structural and functional

74 roles (Bennett et al. 1995; van der Wel 2012). It is believed that the increased interaction area

75 between the protein subunits in the complexes formed via the domain swapping mechanism affects

76 the overall protein stability (Bennett et al. 1994; Liu \& Eisenberg 2002). In some cases, the

77 formation of the quaternary structure of protein by this mechanism is associated with the

78 emergence of new functions in protein oligomers which are not found in the monomeric forms of

79 these proteins (Liu \& Eisenberg 2002). Finally, the domain swapping mechanism is involved in

80 the early stages of the amyloid fibril formation (van der Wel 2012).

81 In this work, to identify which structural features of bOBP are responsible for the formation

82 of its unique dimeric structure and to understand the role of the domain swapping mechanism in

83 maintaining the native structure of the protein, structural properties of the recombinant wild type

84 bOBP and its four mutant forms that cannot dimerize via the domain swapping (Ramoni et al.

85 2008; Ramoni et al. 2002) were analyzed and compared using a spectrum of the biophysical

86 techniques that included intrinsic fluorescence spectroscopy, circular dichroism spectroscopy in

87 the far- and near-UV regions and gel filtration. We also designed two monomeric mutants, GCC-

88 bOBP-W17F and GCC-bOBP-W133F, each containing a single tryptophan residue, for the

89 characterization of the specific features of the microenvironments of these tryptophan residues that

90 affect the intrinsic fluorescence characteristics of this protein. 


\section{MATERIALS AND METHODS}

93 Materials

94 GdnHCl (Nacalai Tesque, Japan) and 1-octen-3-ol (OCT; Sigma-Aldrich, USA) were used 95 without further purification. The protein concentration was $0.1-0.2 \mathrm{mg} / \mathrm{ml}$. The OCT 96 concentration was $10 \mathrm{mM}$. The experiments were performed in $20 \mathrm{mM}$ Na-phosphate-buffered 97 solution at $\mathrm{pH} 7.8$.

98

Gene expression and protein purification

100 The plasmids pT7-7-bOBP which encodes recombinant bOBP and its mutant forms with a 101 poly-histidine tag were used to transform Escherichia coli BL21(DE3) host (Invitrogen) 102 (Stepanenko et al. 2014b). The plasmids encoding bOBP and its mutant forms were purchased 103 from Evrogen JSC. Protein expression was induced by incubating the cells with $0.3 \mathrm{mM}$ of 104 isopropyl-beta-D-1-thiogalactopyranoside (IPTG; Fluka, Switzerland) for $24 \mathrm{~h}$ at $37{ }^{\circ} \mathrm{C}$. The 105 recombinant protein was purified with $\mathrm{Ni}+$-agarose packed in HisGraviTrap columns (GE 106 Healthcare, Sweden). The protein purity was determined through SDS-PAGE in 15\% 107 polyacrylamide gel (Laemmli 1970).

108

109 Analyzing the $3 D$ protein structure

110 We analyzed the microenvironment peculiarities for tryptophan residues in the bOBP and

111 GCC-bOBP structure based on PDB data (Dutta et al. 2009) using the 1OBP (Tegoni et al. 1996)

112 and 2HLV PDB files (Ramoni et al. 2008) as described previously (Giordano et al. 2004;

113 Stepanenko et al. 2014a; Stepanenko et al. 2015; Stepanenko et al. 2012; Turoverov et al. 1985). 
115

116

117

118

119

120

121

122

123

124

125

data were analyzed using the multiexponential approach:

$$
I(t)=\sum_{i} \alpha_{i} \exp \left(-t / \tau_{i}\right)
$$

126

127

where $\alpha_{i}$ and $\tau_{i}$ are amplitude and lifetime of component $i$, respectively, and were $\sum \alpha_{i}=1$. At

128

the same time, more physical sense (Turoverov \& Kuznetsova 1986) is given by the contribution of $i$ component (with $\tau_{i}$ ) $S_{i}$ to the total emission:

$$
S_{i}=\frac{\alpha_{i} \int_{0}^{\infty} \exp \left(-t / \tau_{i}\right) d t}{\sum \alpha_{i} \int_{0}^{\infty} \exp \left(-t / \tau_{i}\right) d t}=\frac{\alpha_{i} \tau_{i}}{\sum \alpha_{i} \tau_{i}} .
$$

The root-mean square value of fluorescent lifetimes, $\langle\tau\rangle$, for biexponential decay was

132 determined as

$$
\langle\tau\rangle=\frac{\alpha_{1} \tau_{1}^{2}+\alpha_{2} \tau_{2}^{2}}{\alpha_{1} \tau_{1}+\alpha_{2} \tau_{2}}=\sum S_{i} \tau_{i}
$$


135 edge $\left(\lambda_{\mathrm{ex}}=297 \mathrm{~nm}\right)$, wherein the tyrosine residue contribution to the bulk protein fluorescence is

136 negligible. The fluorescence spectra position and form were characterized using the parameter

$137 A=I_{320} / I_{365}$, wherein $I_{320}$ and $I_{365}$ are the fluorescence intensities at the emission wavelengths

138320 and $365 \mathrm{~nm}$, respectively (Turoverov \& Kuznetsova 2003). The values for parameter $A$ and

139 the fluorescence spectrum were corrected for instrument sensitivity. The tryptophan fluorescence

140 anisotropy was calculated using the equation:

$141 \quad r=\left(I_{V}^{V}-G I_{H}^{V}\right) /\left(I_{V}^{V}+2 G I_{H}^{V}\right)$,

142 wherein $I_{V}^{V}$ and $I_{H}^{V}$ are the vertical and horizontal fluorescence intensity components upon

143 excitement by vertically polarized light. $G$ is the relationship between the fluorescence intensity

144 vertical and horizontal components upon excitement by horizontally polarized light $\left(G=I_{V}^{H} / I_{H}^{H}\right)$,

$145 \lambda_{\mathrm{em}}=365 \mathrm{~nm}$ (Turoverov et al. 1998).

146 Protein unfolding was initiated by manually mixing the protein solution $(40 \mu \mathrm{L})$ with a buffer

147 solution $(510 \mu \mathrm{L})$ that included the necessary $\mathrm{GdnHCl}$ concentration. The GdnHCl concentration

148 was determined by the refraction coefficient using an Abbe refractometer (LOMO, Russia; (Pace

149 1986)). The dependences of different bOBP fluorescent characteristics on GdnHCl were recorded

150 following protein incubation in a solution with the appropriate denaturant concentration at $4^{\circ} \mathrm{C}$ for

1512,24 and 48 h. bOBP refolding was initiated by diluting the pre-denatured protein (in $3.0 \mathrm{M}$

$152 \mathrm{GdnHCl}, 40 \mu \mathrm{L})$ with the buffer or denaturant solutions at various concentrations $(510 \mu \mathrm{L})$. The

153 spectrofluorometer was equipped with a thermostat that holds the temperature constant at $23^{\circ} \mathrm{C}$.

Circular dichroism measurements

156 The CD spectra were generated using a Jasco-810 spectropolarimeter (Jasco, Japan). Far- 
157 UV CD spectra were recorded in a 1-mm path length cell from $260 \mathrm{~nm}$ to $190 \mathrm{~nm}$ with a $0.1 \mathrm{~nm}$

158 step size. Near-UV CD spectra were recorded in a 10-mm path length cell from $320 \mathrm{~nm}$ to $250 \mathrm{~nm}$

159 with a $0.1 \mathrm{~nm}$ step size. For the spectra, we generated 3 scans on average. The CD spectra for the

160 appropriate buffer solution were recorded and subtracted from the protein spectra.

161

162 Gel filtration experiments

163 We performed gel filtration experiments for bOBP and its mutant forms in a buffered

164 solution without and with addition of GdnHCl using a Superdex-75 PC 3.2/30 column (GE

165 Healthcare, Sweden) and an AKTApurifier system (GE Healthcare, Sweden). The column was

166 equilibrated with the buffered solution or $\mathrm{GdnHCl}$ at the desired concentration, and $10 \mu \mathrm{l}$ of the

167 protein solution prepared under the same conditions was loaded on the pre-equilibrated column.

168 The change in hydrodynamic dimensions for the studied proteins was evaluated as a change in the

169 bOBP or the mutant protein elution volume. Multiple proteins with known molecular masses

170 (aprotinin $(6.5 \mathrm{kDa})$, ribonuclease $(13.7 \mathrm{kDa})$, carbonic anhydrase $(29 \mathrm{kDa})$, ovalbumin $(43 \mathrm{kDa})$

171 and conalbumin $(75 \mathrm{kDa})$, which are chromatography standards from GE Healthcare) were used

172 to generate the calibration curve.

173

174 Evaluation of the intrinsic disorder predisposition

175 The intrinsic disorder propensity of the bOBP was evaluated by several disorder predictors,

176 such as PONDR ${ }^{\circledR}$ VLXT (Dunker et al. 2001), PONDR ${ }^{\circledR}$ VSL2 (Peng et al. 2005), PONDR ${ }^{\circledR}$ VL3 $^{2}$

177 (Peng et al. 2006), and PONDR ${ }^{\circledR}$ FIT (Xue et al. 2010). Effects of the point mutations on the

178 intrinsic disorder predisposition of this protein was analyzed by PONDR ${ }^{\circledR}$ VSL2. In these analyses,

179 scores above 0.5 are considered to correspond to the disordered residues/regions. PONDR ${ }^{\circledR}$ 
180 VSL2B was chosen for the comparative analysis of the bOBP mutants since this tool is one of the

181 more accurate stand-alone disorder predictors (Fan \& Kurgan 2014; Peng \& Kurgan 2012),

182 PONDR $^{\circledR}$ VLXT is known to have high sensitivity to local sequence peculiarities and can be used

183 for identifying disorder-based interaction sites (Stepanenko et al. 2015), PONDR ${ }^{\circledR}$ VL3 provides

184 accurate evaluation of long disordered regions (Turoverov \& Kuznetsova 1986), whereas a

185 metapredictor PONDR-FIT is moderately more accurate than each of the component predictors,

186 PONDR ${ }^{\circledR}$ VLXT (Stepanenko et al. 2015), PONDR ${ }^{\circledR}$ VSL2 (Stepanenko et al. 2014a), PONDR ${ }^{\circledR}$

187 VL3 (Turoverov \& Kuznetsova 1986), FoldIndex (Prilusky et al. 2005), IUPred (Dosztanyi et al.

188 2005), TopIDP (Campen et al. 2008). PONDR-FIT (Tegoni et al. 2000).

189

190 RESULTS AND DISCUSSION

191 It is believed that the introduction of an extra glycine residue after the bOBP residue 121

192 (Figure 2) should result in the increased mobility of the loop connecting $\alpha$-helix and $8^{\text {th }} \beta$-strand

193 of the $\beta$-barrel, which, in its turn, promotes the formation of a monomeric fold of the mutant protein

194 bOBP-Gly121+. Substitutions of the residues Trp64 and His156 to cysteines in bOBP-Gly121+

195 generate a mutant form GCC-bOBP, which should have stable monomeric structure due to the

196 restoration of the disulfide bond typically seeing in classical OBPs. Finally, to characterize specific

197 features of the microenvironments of tryptophan residues W17 and W133 that might affect the

198 intrinsic fluorescence of the protein, we designed two monomeric mutant forms GCC-bOBP-

199 W17F and GCC-bOBP-W133F, each containing a single tryptophan residue.

200 To evaluate potential effects of selected mutations on protein structure, we analyzed 201 substitution-induced changes in the intrinsic disorder propensity of bOBP. It has been pointed out

202 that such computational analysis can provide useful information on the expected outcomes of the 
203 point mutations in proteins (Melnik et al. 2012; Moroz et al. 2013; Uversky et al. 2011; Vacic et 204 al. 2012). Figure 3A represents the results of the computational multi-tool analysis of the per205 residue intrinsic disorder predisposition of bOBP. We used here several members of the PONDR 206 family, PONDR ${ }^{\circledR}$ VLXT (Stepanenko et al. 2015), PONDR ${ }^{\circledR}$ VSL2 (Stepanenko et al. 2014a), and 207 PONDR ${ }^{\circledR}$ FIT (Tegoni et al. 2000). Figure 3A shows that all these tools are generally agree with 208 each other and indicates that although bOBP is predicted to be mostly ordered, this protein 209 possesses several disordered or flexible regions. Disordered regions are defined here as protein 210 fragments containing residues with the disorder scores above the 0.5 threshold, whereas regions 211 are considered flexible if disorder scores of their residues ranges from 0.3 to 0.5 . Figure 3B 212 represents the results of the disorder evaluation in mutant forms of the bOBP and shows the aligned 213 PONDR $^{\circledR}$ VSL2-based disorder profiles for the wild type protein and its four mutants. These 214 analyses revealed that the wild type bOBP and its four mutants are expected to be rather ordered 215 (clearly belonging to the category of hybrid proteins that contain ordered domains and intrinsically 216 disordered regions) and that mutations do not induce significant changes in the bOBP disorder 217 propensity.

218 Previously, we have shown that the recombinant bOBP, unlike native bOBP purified from 219 the tissue, exists in a stable native-like state as a mixture of monomeric and dimeric forms 220 (Stepanenko et al. 2014b) (Table 1). Furthermore, the recombinant bOBP forms dimers in the 221 presence of relatively high denaturant concentrations (e.g., in a solution of $1.5 \mathrm{M}$ guanidine 222 hydrochloride, $\mathrm{GdnHCl})$. The dimerization process is accompanied by the formation of a stable, 223 more compact, intermediate state maximally populated at $0.5 \mathrm{M} \mathrm{GdnHCl}$.

224 In the present work, gel filtration analysis revealed that all investigated mutants of the bOBP, 225 namely bOBP-Gly121+, GCC-bOBP, GCC-bOBP-W17F, and GCC-bOBP-W133F, are 
226 monomers (Figure 4, Table 1). The positions of the elution peaks of the studied mutant bOBP

227 forms coincided with the position of the elution peak of the monomeric form of recombinant

228 bOBP. This allows us to conclude that monomeric forms of mutant proteins bOBP-Gly121+, GCC-

229 bOBP, GCC-bOBP-W17F, and GCC-bOBP-W133F are as compact as the recombinant bOBP

230 monomer. Therefore, the amino acid substitutions introduced to the bOBP sequence did not affect

231 the compact structure of this protein.

232 Investigation of the interaction of recombinant bOBP with its natural ligand 1-Octen-3-ol

233 (OCT) by gel-filtration chromatography revealed that the elution profile of the bOBP/OCT

234 complex contained two peaks (Figure 4). These data indicate that similar to the recombinant bOBP

235 the bOBP/OCT complex exists as a mixture of monomeric and dimeric forms of the protein.

236 However, the positions of the two peaks in the bOBP/OCT elution profile are shifted to slightly

237 higher elution volume, suggesting that the OCT binding induces partial compaction of both the

238 monomeric and dimeric forms of the protein. Evaluated hydrodynamic dimensions of the bOBP

239 and its complex with ligand confirmed this assumption (Table 1). Furthermore, the complexed

240 forms of the bOBP mutants were also more compact than their OCT-free forms (Table 1). The

241 similar behavior of recombinant bOBP and its mutants suggested that the introduced mutations do

242 not affect functional activity of the bOBP and the ability of this protein to bind a natural ligand.

243 Table 1 and Figure 5 shows that the recombinant bOBP is characterized by a relatively long-

244 wave position of the intrinsic tryptophan fluorescence $\left(\lambda_{\max }=335 \mathrm{~nm}\right.$ at $\left.\lambda_{\mathrm{ex}}=297 \mathrm{~nm}\right)$. Although

245 in comparison of the position of the emission spectrum of completely solvent accessible $\operatorname{Trp}\left(\lambda_{\max }\right.$

$246=350 \mathrm{~nm}$ ), the indicated value of $335 \mathrm{~nm}$ found for the recombinant bOBP is noticeably blue-

247 shifted, this value is definitely too far from a short wavelength position of tryptophan fluorescence

248 found in some proteins. In fact, one of the shortest wavelength position of Trp fluorescence $\left(\lambda_{\max }\right.$ 
$249=308 \mathrm{~nm}$ ) was described for the blue copper protein azurine from Pseudomonas aeruginosa

250 (Turoverov et al. 1985), and a close short wavelength fluorescence spectra with $\lambda_{\max }=312 \mathrm{~nm}$ and

$251318 \mathrm{~nm}$ were also described for the DsbC from E.coli (Stepanenko et al. 2004) and for another

252 cooper-containing bacterial protein amicyanin from Thiobacillus versutus (Rosato et al. 1991), 253 respectively.

254 The intrinsic fluorescence of bOBP is determined by three tryptophan residues, two of which 255 are located in the $\beta$-sheet (Trp17 is in the first $\beta$-strand, and Trp64 is in the fourth $\beta$-strand), 256 whereas Trp133 is included into a single $\alpha$-helix of this protein. Among all the tryptophan residues 257 of the protein Trp133 has the lowest density of the microenvironment $(\mathrm{d}=0.54)$, indicating that it 258 is partially accessible to the solvent (Table 2). The microenvironments of Trp17 and Trp64 are 259 more dense ( 0.80 and 0.71 , respectively), but also more polar compared with the Trp133 local 260 environment (Tables 2-5).

261 It should be noted that the side chains of the charged residues Lys121 and Lys59 included in 262 the microenvironments of Trp17 and Trp64, respectively, are oriented parallel to the indole ring 263 of the corresponding tryptophan residue, and their NZ amino groups are located at a short distance 264 from NE1 group of the corresponding tryptophan residue (5.16 and $4.55 \AA$ for NZ groups Lys121 265 and Lys59, Tables 3-4). Therefore, the presence of a partial fluorescence quenching of Trp17 and 266 Trp64 cannot be excluded, since fluorescence quenching was previously reported for a single 267 tryptophan residue Trp16 of porcine OBP that has similar features in its microenvironment 268 (Staiano et al. 2007; Stepanenko et al. 2008).

269 Recombinant bOBP is characterized by high values of fluorescence anisotropy and 270 fluorescence lifetime (Table 1), and also has a pronounced CD spectrum in the near-UV region 
271 (Figure 6). These observations indicate that the environment of tryptophan residues of this protein

272 is quite rigid and asymmetric.

273 The monomeric bOBP-Gly121+ is characterized by a somewhat longer wavelength of the

274 tryptophan fluorescence spectrum and lower value of the fluorescence anisotropy compared to the

275 recombinant bOBP (Table 1, Figure 5). The near-UV CD spectrum of the bOBP-Gly121+ is almost

276 indistinguishable for the spectrum of recombinant bOBP (Figure 6). This indicates that although

277 the overall spatial structure of the protein is not perturbed by adding an extra Gly residue after the

278 position 121 , the local microenvironment of the tryptophan residues become less dense due to this

279 sequence perturbation. Importantly, the magnitudes of the fluorescence lifetime and fluorescence

280 quantum yield of the bOBP-Gly121+ are higher than those for the recombinant bOBP. It is likely

281 that the more mobile microenvironments of the tryptophan residues in bOBP-Gly121+ might result

282 in the removal of some potential quenching groups from the indole ring of these tryptophan

283 residues, thereby leading to a weakening of the quenching effects.

284 The values of the fluorescence parameters such as the position of the maximal tryptophan

285 fluorescence, fluorescence anisotropy, and fluorescence lifetime for the triple mutant GCC-bOBP,

286 which was designed to have disulfide bond via substituting residues Trp64 and His156 of the

287 bOBP-Gly121+ to the cysteine residues, were similar to these parameters recorded for the

288 recombinant bOBP (Table 1, Figure 5). The intensity of the negative band in the near-UV CD

289 spectrum of the GCC-bOBP variant was greater than that of the recombinant bOBP (Figure 6).

290 These data demonstrate the stabilizing effect of the disulfide bond to the protein structure. The

291 intensity of the GCC-bOBP tryptophan fluorescence was approximately $25 \%$ lower than that of

292 the recombinant bOBP (Figure 5). Since the structure of the GCC-bOBP retained only two of the 
293 three tryptophan residues of the protein, namely Trp17 and Trp133, it can be argued that the

294 removed residue Trp64 made a significant contribution to the fluorescence of recombinant bOBP.

295 Mutant forms designed to have a single tryptophan residue, GCC-bOBP-W17F (contains

296 only Trp133) and the GCC-bOBP-W133F (contains only Trp17) are characterized by the

297 substantially different parameters of tryptophan fluorescence and near-UV CD spectra (Figures 5

298 and 6). GCC-bOBP-W17F has the most long-wavelength fluorescence spectrum among all

299 proteins studied here, the lowest values of the parameter $A$ and the fluorescence anisotropy $r$,

300 whereas GCC-bOBP-W133F has the most short-wavelength fluorescence spectrum and the

301 highest values of the parameter $A$ and fluorescence anisotropy $r$ (Table 1, Figure 5). GCC-bOBP-

302 W133F is also characterized by the most intense and short wavelength near-UV CD spectrum. At

303 the same time, the near UV-CD spectrum of the GCC-bOBP-W17F is less intense and the most

304 long wavelength among all proteins analyzed in this study (Figures 6). These data indicate that the

305 microenvironments of the residues Trp17 and Trp133 are significantly different from each other.

306 It should be noted that the calculated total spectrum of the intrinsic fluorescence of these two

307 proteins GCC-bOBP-W17F and GCC-bOBP-W133F (calculated as a weighted sum of individual

308 spectra) coincides with the tryptophan fluorescence spectrum of GCC-bOBP (Figure 5). These

309 data together with the results of the gel filtration analysis suggested that the mutant proteins GCC-

310 bOBP-W17F and GCC-bOBP-W133F maintained native-like, mostly unperturbed spatial

311 structures, and that the microenvironments of their residues $\operatorname{Trp} 17$ and $\operatorname{Trp} 133$ are similar to the

312 environments of these residues in the GCC-bOBP protein.

313 The position of the tryptophan fluorescence spectrum of the GCC-bOBP-W17F mutant, and

314 the values of its parameter $A$, fluorescence anisotropy, and fluorescence lifetime suggest that the

315 microenvironment of the Trp133 residue is relatively polar and mobile, and the residue itself 
316 contributes significantly to the total fluorescence of this protein (Table 1). These data agree well

317 with the results of the analysis of the specific characteristics of the microenvironment of

318 tryptophan residues in the wild type bOBP (Tables 2-5) and the GCC-bOBP mutant (Tables 2-5).

319 At the same time, the spectral characteristics of the GCC-bOBP-W133F mutant suggest that the

$320 \operatorname{Trp} 17$ can be considered as an internal residue located within a very dense, inaccessible to solvent

321 microenvironment. Furthermore, the fluorescence of this residue is substantially quenched (Table

322 1, Figure 5).

323 Analysis of the microenvironment of tryptophan residue Trp17 in the wild type bOBP and

324 its GCC-bOBP mutant (Table 3) shows that it is rather similar to the environment of Trp16 in

325 pOBP, which was considered to be quenched by Lys120 (Staiano et al. 2007). However, there are

326 some differences in the conformation of Lys side chain in these proteins. In pOBP, the side chain

327 of Lys120 is located practically parallel to the indole ring of Trp16, and the nearest atom of this

328 residue NZ is separated from the center of the indole ring by only $4.15 \AA$. This conformation of

329 Lys120 could be stabilized by H-bond formation between the $\mathrm{O}$ atom of main chain of Lys 15 and

$330 \mathrm{NZ}$ atom of Lys120 (2.63 A, Figure 7). In bOBP, the side chain of the Lys121 is extended and

331 oriented in such a way that its NZ atom is located far from the center of the indole ring of Trp17.

332 Therefore, it is unlikely that the Trp17 of bOBP is quenched by the Lys121. At the same time,

333 Trp17 of bOBP can be quenched by electron transfer to the amide groups of the main chain. This

334 mechanism works if there are negatively charged groups in the near vicinity of the indole ring and

335 there are positively charged groups near the amides (Callis 2014; Scott \& Callis 2013). In the case

336 of Trp17 of bOBP, such situation is provided by Arg 18 with its $\mathrm{NH} 1$ atom being H-bonded to the

337 amide of the tryptophan residue ( $2.95 \AA$ ) and by Leu 13 with its main chain $\mathrm{O}$ atom being H-bonded

338 to the indole ring HN ( $2.87 \AA)$. The Trp64 of bOBP makes a significant contribution to the total 
339 fluorescence of the recombinant bOBP. Indeed, in the microenvironment of Trp64 there are no

340 groups which could promote electron transfer to amide groups of the main chain (Table 4).

341 Additionally, the microenvironment of this Trp residue contains only a lysine residue (Lys59) in

342 the conformation similar to that of Lys121, which does not favor fluorescence quenching.

343 The far-UV CD spectra recorded for the recombinant bOBP and its four mutant forms

344 (bOBP-Gly121+, GCC-bOBP, GCC-bOBP-W17F, and GCC-bOBP-W133F) are rather similar

345 and have a shape characteristic of protein enriched in the $\beta$-structural elements (Figure 8).

346 Decomposition of the far-UV CD spectrum of the recombinant bOBP using the Provencher's

347 algorithm (Provencher \& Glockner 1981) revealed that this protein contains 13\% $\alpha$-helix, $36 \% \beta$ -

348 sheet, and $20 \% \beta$-turns (Table 6). These data agree well with the results of the X-ray analysis of

349 the wild type bOBP ( $13 \% \alpha$-helix and $46 \% \beta$-sheets). In the bOBP-Gly121+, adding the Gly $121+$

350 insert leads to a certain decrease in the content of $\alpha$-helical elements (Table 6). Obviously, the

351 insertion of an extra Gly121 residue to the loop segment preceding the single $\alpha$-helix of the protein

352 reduces the length of this helical element. On the other hand, introduction of a disulfide bond to

353 the structure of the triple mutant GCC-bOBP leads to a full recovery of the protein secondary

354 structure. Since the introduced disulfide bridge tightens the $\alpha$-helical region to the $\beta$-barrel frame

355 of the protein, this tightened overall fold may be the reason for the restoration of the bOBP

356 secondary structure. These data also confirm the stabilizing effects of the disulfide bond in the

357 monomeric form of the protein. Replacement of the tryptophan residue Trp133 to phenylalanine

358 in the mutant form GCC-bOBP-W133F leads to an increase in the content of $\alpha$-helical elements

359 while the content of $\beta$-sheets stays unaltered. Instead, the amount of unordered structure is lowered.

360 It is likely that the substitution of the tryptophan residue with a bulky side chain by a less massive

361 residue in the single $\alpha$-helix of this protein may leads to prolongation of the helical element 
362 reducing some steric constraints inside the $\alpha$-helix. In contrast, in the GCC-bOBP-W17F mutant,

363 replacement of a tryptophan residue Trp17 to phenylalanine may indicate a marked decrease in $\alpha$ -

364 helical structure and marked increase in $\beta$-structure with no changes in unordered structure content.

365 We hypothesize that the absence of the bulky side chain of Trp17 in the first $\beta$-strand of the $\beta$ -

366 barrel may favor the formation of a closer contact between the first and the ninth $\beta$-strands of the

367 protein thus stabilizing $\beta$-barrel.

368 In the presence of the OCT ligand, the fluorescent characteristics and the near- and far-UV

369 CD spectra of the recombinant bOBP and its mutant forms undergo significant changes, indicating

370 compaction and stabilization of the spatial structure of these proteins (Tables 1 and 6). These data

371 also suggest that all mutant forms of bOBP analyzed in this work retain the ability to bind a natural

372 ligand.

373 Therefore, all mutant forms of bOBP generally retain tertiary and secondary structure. Their

374 structural organization is rather similar to that of the recombinant bOBP. The structure of the GCC-

375 bOBP is closest to the structure of the recombinant wild type bOBP. The observed changes in the

376 local structure of the mutant forms of bOBP do not violate the ability of the protein to correctly

377 fold and bind a natural ligand. Ligand binding to the bOBP mutant forms leads to a more compact

378 state of the studied proteins and does not alter its oligomeric status.

379

380 CONCLUSIONS

381 We show here that the insertion of Gly121+ leads to disruption of the domain swapping

382 mechanism, resulting in a stable monomeric mutant protein bOBP-Gly121+. The introduction of

383 a disulfide bond induces noticeable stabilization of the monomeric fold of the GCC-bOBP mutant.

384 The amino acid substitutions introduced to bOBP in this study, such as Gly121+ insertion in the 
385 bOBP-Gly121+ mutant, replacement of the Trp64 and His156 to the cysteine residues in the GCC-

386 bOBP mutant, and replacement of the Trp17 and Trp133 residues to phenylalanine in the GCC-

387 bOBP-W17F and GCC-bOBP-W133F mutants, do not disrupt the functional activity of the

388 protein. We show that the ligand binding leads to the formation of a more compact and stable state

389 of the recombinant bOBP and its mutant monomeric forms. We also describe the peculiarities of

390 the microenvironment of tryptophan residues of the protein which are essential for the formation

391 of the fluorescent properties of the protein and which were not described previously.

392 


\section{FIGURE LEGENDS}

395

396 Figure 1. 3D structure of bOBP. The individual subunits in the protein are in gray and orange.

397 The tryptophan residues in the different subunits are indicated in red and blue. The Lys 121 residue

398 after which an extra glycine residue are inserted in the mutant form bOBP-Gly121+ is shown in

399 green. Additionally the residues Trp 64 and His 156 (yellow) are substituted for cysteine in the

400 mutant form GCC-bOBP. The drawing was generated based on the 1OBP file (Tegoni et al. 1996)

401 from PDB (Dutta et al. 2009) using the graphic software VMD (Hsin et al. 2008) and Raster3D

402 (Merritt \& Bacon 1977).

403

404 Figure 2. The comparison of the primary sequence for the recombinant bOBPwt and its mutant 405 forms bOBP-Gly121+ and GCC- bOBP, which are not able to form domain-swapped dimers. The 406 mutant forms GCC-bOBP-W17F and GCC-bOBP-W133F designed to contain a single tryptophan 407 residue were produced to investigate the peculiarities of the microenvironment of the tryptophan 408 residues.

409

410 Figure 3. Intrinsic disorder propensity of the wild type bOBP and its mutants. A. Per-residue

411 disorder propensity of the wild type bOBP evaluated by members of the PONDR family, PONDR ${ }^{\circledR}$

412 VLXT (Dunker et al. 2001) (green line), PONDR ${ }^{\circledR}$ VSL2 (Peng et al. 2005) (blue line), PONDR ${ }^{\circledR}$

413 FIT (Xue et al. 2010)(red line) and PONDR ${ }^{\circledR}$ VL3 (Peng et al. 2006) (pink line). Localization of

414 known elements of the bOBP secondary structure is shown by colored bars at the bottom of the 415 plot. Light pink shadow around the PONDR ${ }^{\circledR}$ FIT curve represents distribution of errors in the 
416 disorder score evaluation. B. Effects of mutations on the intrinsic disorder propensity of bOBP

417 evaluated by PONDR ${ }^{\circledR}$ VSL2.

418

419 Figure 4. Hydrodynamic characteristics of the bOBP and its mutants. The changes of 420 hydrodynamic dimensions of recombinant bOBP (1) and its mutant forms bOBP-Gly121+ (2), 421 GCC-bOBP (3), GCC-bOBP-W17F (4) and GCC-bOBP-W133F (5) in the absence (solid lines) 422 and the presence of OCT (dotted lines).

424 Figure 5. Tertiary structure changes for bOBP (red) and its mutant forms bOBP-Gly121+ 425 (green), GCC-bOBP (blue), GCC-bOBP-W17F (gray) and GCC-bOBP-W133F (dark 426 yellow) in different structural states are indicated by intrinsic tryptophan fluorescence $\left(\lambda_{\text {ex }}=297\right.$ 427 $\mathrm{nm}$ ). The spectra shown are for the protein in buffered solution (solid line), in the presence of natural ligand OCT (dotted line) and in the presence of $3.5 \mathrm{M} \mathrm{GdnHCl}$ (dashed line). The corresponding spectra in light blue were obtained as a sum of the spectra for GCC-bOBP-W17F and GCC-bOBP-W133F.

431

432

Figure 6. Tertiary structure for bOBP (red) and its mutant forms bOBP-Gly121+ (green), GCC-bOBP (blue), GCC-bOBP-W17F (gray) and GCC-bOBP-W133F (dark yellow) in buffered solution are indicated by near-UV CD spectra. The spectrum in light blue was obtained as a sum of the spectra for GCC-bOBP-W17F and GCC-bOBP-W133F.

Figure 7. The microenvironment of Trp17 in bOBP (A) and Trp16 in pOBP (B). The spatial 
439 the protein core is shown as transparent structure. The drawing was generated based on the 1OBP 440 file (Tegoni et al. 1996) and 1A3Y (Spinelli et al., 1998) from PDB (Dutta et al. 2009) using the 441 graphic software VMD (Hsin et al. 2008) and Raster3D (Merritt \& Bacon 1977).

442

443

444

445

446

447

448

449

450

451

452

453

454

455

456

457

458

459

460

461

462

463

464

465

466

467

468

469

470

471

472

473

474

475

Figure 8. Secondary structure for bOBP (red) and its mutant forms bOBP-Gly121+ (green), GCC-bOBP (blue), GCC-bOBP-W17F (gray) and GCC-bOBP-W133F (dark yellow) in buffered solution are indicated by far-UV CD spectra.

\section{REFERENCES}

Bennett MJ, Choe S, and Eisenberg D. 1994. Domain swapping: entangling alliances between proteins. Proc Natl Acad Sci U S A 91:3127-3131.

Bennett MJ, Schlunegger MP, and Eisenberg D. 1995. 3D domain swapping: a mechanism for oligomer assembly. Protein Sci 4:2455-2468.

Bianchet MA, Bains G, Pelosi P, Pevsner J, Snyder SH, Monaco HL, and Amzel LM. 1996. The three-dimensional structure of bovine odorant binding protein and its mechanism of odor recognition. Nat Struct Biol 3:934-939.

Callis PR. 2014. Binding phenomena and fluorescence quenching. II: Photophysics of aromatic residues and dependence of fluorescence spectra on protein

conformation. Journal of Molecular Structure 1077:22-29.

Campen A, Williams RM, Brown CJ, Meng J, Uversky VN, and Dunker AK. 2008. TOP-IDPscale: a new amino acid scale measuring propensity for intrinsic disorder. Protein Pept Lett 15:956-963. 10.2174/092986608785849164

Dosztanyi Z, Csizmok V, Tompa P, and Simon I. 2005. IUPred: web server for the prediction of intrinsically unstructured regions of proteins based on estimated energy content. Bioinformatics 21:3433-3434. 10.1093/bioinformatics/bti541

Dunker AK, Lawson JD, Brown CJ, Williams RM, Romero P, Oh JS, Oldfield CJ, Campen AM, Ratliff CM, Hipps KW, Ausio J, Nissen MS, Reeves R, Kang C, Kissinger CR, Bailey RW, Griswold MD, Chiu W, Garner EC, and Obradovic Z. 2001. Intrinsically disordered protein. J Mol Graph Model 19:26-59. 10.1016/S1093-3263(00)00138-8

Dutta S, Burkhardt K, Young J, Swaminathan GJ, Matsuura T, Henrick K, Nakamura H, and Berman HM. 2009. Data deposition and annotation at the worldwide protein data bank. Mol Biotechnol 42:1-13.

Fan X, and Kurgan L. 2014. Accurate prediction of disorder in protein chains with a comprehensive and empirically designed consensus. J Biomol Struct Dyn 32:448-464. 10.1080/07391102.2013.775969

Flower DR, North AC, and Sansom CE. 2000. The lipocalin protein family: structural and sequence overview. Biochim Biophys Acta 1482:9-24. 
476

477

478

479

480

481

482

483

484

485

486

487

488

489

490

491

492

493

494

495

496

497

498

499

500

501

502

503

504

505

506

507

508

509

510

511

512

513

514

515

516

517

518

519

520

Fonin AV, Sulatskaya AI, Kuznetsova IM, and Turoverov KK. 2014. Fluorescence of dyes in solutions with high absorbance. Inner filter effect correction. PLoS One 9:e103878. 10.1371/journal.pone.0103878

Giordano A, Russo C, Raia CA, Kuznetsova IM, Stepanenko OV, and Turoverov KK. 2004. Highly UV-absorbing complex in selenomethionine-substituted alcohol dehydrogenase from Sulfolobus solfataricus. J Proteome Res 3:613-620.

Hsin J, Arkhipov A, Yin Y, Stone JE, and Schulten K. 2008. Using VMD: an introductory tutorial. Curr Protoc Bioinformatics Chapter 5:Unit 57.

Laemmli UK. 1970. Cleavage of structural proteins during the assembly of the head of bacteriophage T4. Nature 227:680-685.

Liu Y, and Eisenberg D. 2002. 3D domain swapping: as domains continue to swap. Protein Sci 11:1285-1299.

Melnik BS, Povarnitsyna TV, Glukhov AS, Melnik TN, Uversky VN, and Sarma RH. 2012. SSStabilizing Proteins Rationally: Intrinsic Disorder-Based Design of Stabilizing Disulphide Bridges in GFP. J Biomol Struct Dyn 29:815-824. 10.1080/07391102.2012.10507414

Merritt EA, and Bacon DJ. 1977. Raster3D: Photorealistic molecular graphics. . Methods enzymol 277:505-524.

Moroz NA, Novak SM, Azevedo R, Colpan M, Uversky VN, Gregorio CC, and Kostyukova AS. 2013. Alteration of tropomyosin-binding properties of tropomodulin-1 affects its capping ability and localization in skeletal myocytes. $J$ Biol Chem 288:4899-4907. 10.1074/jbc.M112.434522

Pace CN. 1986. Determination and analysis of urea and guanidine hydrochloride denaturation curves. Methods Enzymol 131:266-280.

Peng K, Radivojac P, Vucetic S, Dunker AK, and Obradovic Z. 2006. Length-dependent prediction of protein intrinsic disorder. BMC Bioinformatics 7:208. 10.1186/1471-2105-7-208

Peng K, Vucetic S, Radivojac P, Brown CJ, Dunker AK, and Obradovic Z. 2005. Optimizing long intrinsic disorder predictors with protein evolutionary information. J Bioinform Comput Biol 3:35-60. 10.1142/S0219720005000886

Peng ZL, and Kurgan L. 2012. Comprehensive comparative assessment of in-silico predictors of disordered regions. Curr Protein Pept Sci 13:6-18. 10.2174/138920312799277938

Prilusky J, Felder CE, Zeev-Ben-Mordehai T, Rydberg EH, Man O, Beckmann JS, Silman I, and Sussman JL. 2005. FoldIndex: a simple tool to predict whether a given protein sequence is intrinsically unfolded. Bioinformatics 21:3435-3438. 10.1093/bioinformatics/bti537

Provencher SW, and Glockner J. 1981. Estimation of globular protein secondary structure from circular dichroism. Biochemistry 20:33-37.

Ramoni R, Spinelli S, Grolli S, Conti V, Merli E, Cambillau C, and Tegoni M. 2008. Deswapping bovine odorant binding protein. Biochim Biophys Acta 1784:651-657.

Ramoni R, Vincent F, Ashcroft AE, Accornero P, Grolli S, Valencia C, Tegoni M, and Cambillau C. 2002. Control of domain swapping in bovine odorant-binding protein. Biochem $\mathrm{J}$ 365:739-748.

Rosato N, Mei G, Savini I, Del Bolgia F, Finazzi-Agro A, Lommen A, and Canters GW. 1991. Intrinsic fluorescence of the bacterial copper-containing protein amicyanin. Arch Biochem Biophys 284:112-115.

Scott JN, and Callis PR. 2013. Insensitivity of tryptophan fluorescence to local charge mutations. J Phys Chem B 117:9598-9605. 10.1021/jp4041716 
521

522

523

524

525

526

527

528

529

530

531

532

533

534

535

536

537

538

539

540

541

542

543

544

545

546

547

548

549

550

551

552

553

554

555

556

557

558

559

560

561

562

563

564

565

Spinelli S, Ramoni R, Grolli S, Bonicel J, Cambillau C, and Tegoni M. 1998. The structure of the monomeric porcine odorant binding protein sheds light on the domain swapping mechanism. Biochemistry 37:7913-7918.

Staiano M, D'Auria S, Varriale A, Rossi M, Marabotti A, Fini C, Stepanenko OV, Kuznetsova IM, and Turoverov KK. 2007. Stability and dynamics of the porcine odorant-binding protein. Biochemistry 46:11120-11127.

Stepanenko OV, Bublikov GS, Stepanenko OV, Shcherbakova DM, Verkhusha VV, Turoverov KK, and Kuznetsova IM. 2014a. A knot in the protein structure - probing the near-infrared fluorescent protein iRFP designed from a bacterial phytochrome. Febs J 281:2284-2298. 10.1111/febs. 12781

Stepanenko OV, Fonin AV, Stepanenko OV, Staiano M, D'Auria S, Kuznetsova IM, and Turoverov KK. 2015. Tryptophan residue of the D-galactose/D-glucose-binding protein from E. Coli localized in its active center does not contribute to the change in intrinsic fluorescence upon glucose binding. J Fluoresc 25:87-94. 10.1007/s10895-014-1483-z

Stepanenko OV, Kuznetsova IM, Turoverov KK, Huang C, and Wang CC. 2004. Conformational change of the dimeric DsbC molecule induced by $\mathrm{GdnHCl}$. A study by intrinsic fluorescence. Biochemistry 43:5296-5303.

Stepanenko OV, Marabotti A, Kuznetsova IM, Turoverov KK, Fini C, Varriale A, Staiano M, Rossi M, and D'Auria S. 2008. Hydrophobic interactions and ionic networks play an important role in thermal stability and denaturation mechanism of the porcine odorantbinding protein. Proteins 71:35-44.

Stepanenko OV, Stepanenko OV, Kuznetsova IM, Shcherbakova DM, Verkhusha VV, and Turoverov KK. 2012. Distinct effects of guanidine thiocyanate on the structure of superfolder GFP. PLoS One 7:e48809.

Stepanenko OV, Stepanenko OV, Staiano M, Kuznetsova IM, Turoverov KK, and D'Auria S. 2014b. The quaternary structure of the recombinant bovine odorant-binding protein is modulated by chemical denaturants. PLoS One 9:e85169. 10.1371/journal.pone.0085169

Tegoni M, Pelosi P, Vincent F, Spinelli S, Campanacci V, Grolli S, Ramoni R, and Cambillau C. 2000. Mammalian odorant binding proteins. Biochim Biophys Acta 1482:229-240.

Tegoni M, Ramoni R, Bignetti E, Spinelli S, and Cambillau C. 1996. Domain swapping creates a third putative combining site in bovine odorant binding protein dimer. Nat Struct Biol 3:863-867.

Turoverov KK, Biktashev AG, Dorofeiuk AV, and Kuznetsova IM. 1998. [A complex of apparatus and programs for the measurement of spectral, polarization and kinetic characteristics of fluorescence in solution]. Tsitologiia 40:806-817.

Turoverov KK, and Kuznetsova IM. 1986. What causes the variation of polarization degree across the emission spectrum of proteins? Biophys Chem 24:327-335. 0301-4622(86)85038-4 [pii]

Turoverov KK, and Kuznetsova IM. 2003. Intrinsic fluorescence of actin. J Fluorescence 13:4157.

Turoverov KK, Kuznetsova IM, and Zaitsev VN. 1985. The environment of the tryptophan residue in Pseudomonas aeruginosa azurin and its fluorescence properties. Biophys Chem 23:7989.

Uversky VN, Shah SP, Gritsyna Y, Hitchcock-DeGregori SE, and Kostyukova AS. 2011. Systematic analysis of tropomodulin/tropomyosin interactions uncovers fine-tuned binding 
566 specificity of intrinsically disordered proteins. $J$ Mol Recognit 24:647-655. $567 \quad 10.1002 / \mathrm{jmr} .1093$

568 Vacic V, Markwick PR, Oldfield CJ, Zhao X, Haynes C, Uversky VN, and Iakoucheva LM. 2012. 569 Disease-associated mutations disrupt functionally important regions of intrinsic protein 570 disorder. PLoS Comput Biol 8:e1002709. 10.1371/journal.pcbi.1002709 van der Wel PC. 2012. Domain swapping and amyloid fibril conformation. Prion 6:211-216.

572 Xue B, Dunbrack RL, Williams RW, Dunker AK, and Uversky VN. 2010. PONDR-FIT: a meta573 predictor of intrinsically disordered amino acids. Biochim Biophys Acta 1804:996-1010. 10.1016/j.bbapap.2010.01.011 


\section{Table $\mathbf{1}$ (on next page)}

Table 1 . Characteristics of recombinant bOBPwt and its mutant forms indifferent structural states as well as in the presence of natural ligand OCT. 
1 Table 1. Characteristics of recombinant bOBPwt and its mutant forms in different structural 2 states as well as in the presence of natural ligand OCT.

3

\begin{tabular}{|c|c|c|c|c|c|c|}
\hline & \multicolumn{4}{|c|}{ Intrinsic fluorescence } & \multicolumn{2}{|c|}{$\begin{array}{c}\text { Hydrodynamic } \\
\text { dimensions }\end{array}$} \\
\hline & $\begin{array}{c}\lambda_{\max } \\
\mathrm{nm}^{1}\end{array}$ & $\begin{array}{c}\text { Parameter } \\
A^{1}\end{array}$ & $r^{2}$ & $\begin{array}{l}<\tau>, \mathrm{ns}^{3} \\
S_{i} ; \tau_{i}(\mathrm{~ns})\end{array}$ & $\begin{array}{l}1 \text { peak, } \\
\text { kDa }\end{array}$ & $\begin{array}{c}2 \text { peak, } \\
\text { kDa }\end{array}$ \\
\hline $\begin{array}{l}\text { bOBPwt } \\
\text { (in buffered solution) }^{4}\end{array}$ & 335 & 1.21 & 0.170 & $\begin{array}{l}4.4 \pm 0.2: \\
0.45 ; 2.7 \\
0.55 ; 6.0\end{array}$ & 43.9 & 23.8 \\
\hline $\begin{array}{l}\text { bOBPwt } \\
\left(\text { in } 0.5 \mathrm{M} \mathrm{GdnHCl}-I_{1} \text { state }\right)^{4}\end{array}$ & 337 & 1.10 & 0.166 & $\begin{array}{l}4.6 \pm 0.2: \\
0.30 ; 2.6 \\
0.70 ; 5.3\end{array}$ & 34.0 & 19.3 \\
\hline $\begin{array}{l}\text { bOBPwt } \\
\left(\text { in } 1.6 \mathrm{M} \mathrm{GdnHCl}-I_{2} \text { state }\right)^{4}\end{array}$ & 335 & 1.20 & 0.180 & $\begin{array}{l}4.8 \pm 0.1: \\
0.43 ; 2.5 \\
0.57 ; 6.4\end{array}$ & 43.6 & \\
\hline bOBPwt/OCT & 334 & 1.30 & 0.177 & & 39.6 & 21.5 \\
\hline bOBPwt in $3.5 \mathrm{M} \mathrm{GdnHCl}$ & 349 & 0.47 & 0.062 & & & \\
\hline bOBP/Gly121+ & 336 & 1.13 & 0.166 & $\begin{array}{l}4.6 \pm 0.1: \\
0.14 ; 1.6 \\
0.86 ; 5.1\end{array}$ & 23.6 & \\
\hline bOBP/Gly121+/OCT & 335 & 1.12 & 0.170 & & 21.5 & \\
\hline bOBP/Gly121+ in $3.5 \mathrm{M} \mathrm{GdnHCl}$ & 350 & 0.47 & 0.059 & & & \\
\hline GCC-bOBP & 335 & 1.05 & 0.170 & $\begin{array}{l}4.3 \pm 0.2: \\
0.45 ; 2.9 \\
0.55 ; 5.4\end{array}$ & 23.6 & \\
\hline GCC-bOBP/OCT & 335 & 1.05 & 0.174 & & 22.5 & \\
\hline GCC-bOBP in $3.5 \mathrm{M} \mathrm{GdnHCl}$ & 348 & 0.48 & 0.060 & & & \\
\hline GCC-bOBP-W17F & 339 & 0.82 & 0.164 & $\begin{array}{l}4.7 \pm 0.1: \\
0.75 ; 3.8 \\
0.25 ; 7.1\end{array}$ & 23.6 & \\
\hline GCC-bOBP-W17F/OCT & 339 & 0.82 & 0.165 & & 22.5 & \\
\hline GCC-bOBP-W17F in $3.5 \mathrm{M} \mathrm{GdnHCl}$ & 349 & 0.49 & 0.066 & & & \\
\hline GCC-bOBP-W133F & 325 & 2.83 & 0.186 & $\begin{array}{c}1.9 \pm 0.4: \\
0.73 ; 0.20 \\
0.27 ; 4.3\end{array}$ & 23.6 & \\
\hline GCC-bOBP-W133F/OCT & 323 & 3.02 & 0.189 & & 23.6 & \\
\hline GCC-bOBP-W133F in $3.5 \mathrm{M} \mathrm{GdnHCl}$ & 350 & 0.46 & 0.056 & & & \\
\hline
\end{tabular}

4

$5 \quad{ }^{1} \lambda_{\text {ex }}=297 \mathrm{~nm}$;

$6 \quad 2 \lambda_{\mathrm{ex}}=297 \mathrm{~nm}, \lambda_{\mathrm{em}}=365 \mathrm{~nm}$;

$7 \quad{ }^{3} \lambda_{\mathrm{ex}}=297 \mathrm{~nm}, \lambda_{\mathrm{em}}=335 \mathrm{~nm}$

$8{ }^{4}$ The data are from (Stepanenko et al. 2014b)

9

10 
Table 2 (on next page)

Table 2. Side chain conformation of Trp residues in bOBPwtand GCC-bOBP 
1

2

3

4

5

6

7

\begin{tabular}{c|c|ccc}
\hline Protein & Residue & $\mathrm{N}(d)^{*}$ & $\chi_{1},(\mathrm{deg})^{*}$ & $\chi_{2},(\mathrm{deg})^{*}$ \\
\hline bOBPwt & Trp 17 & $84(0.80)$ & 283.18 & 78.87 \\
& Trp 64 & $80(0.71)$ & 278.04 & 99.85 \\
& Trp 133 & $56(0.54)$ & 287.95 & 112.22 \\
\hline GCC-bOBP & Trp 17 & $87(0.83)$ & 292.40 & 82.09 \\
& $\operatorname{Trp~133~}$ & $48(0.50)$ & 283.53 & 103.91 \\
\hline
\end{tabular}

$8 * \mathrm{~N}$ is the number of atoms in the microenvironment of tryptophan residue; $d$ is the density of

9 tryptophan residue microenvironment; $\chi_{1}$ and $\chi_{2}$ are the angles characterizing the conformation of

10 tryptophan residue side chain. 


\section{Table 3(on next page)}

Table 3 . Characteristics of the Trp 17 microenvironment inbOBPwt and GCC-bOBP 
1 Table 3. Characteristics of the Trp 17 microenvironment in bOBPwt and GCC-bOBP 2

\begin{tabular}{|c|c|c|c|c|c|}
\hline \multicolumn{3}{|c|}{ bOBPwt } & \multicolumn{3}{|c|}{ GCC-bOBP } \\
\hline $\begin{array}{c}\text { atoms of the } \\
\text { microenvironment }\end{array}$ & $\begin{array}{c}\text { atoms of } \\
\text { TRP }\end{array}$ & $\mathrm{R}^{*}, \AA$ & $\begin{array}{c}\text { atoms of } \\
\text { microenvironment }\end{array}$ & $\begin{array}{c}\text { atoms of } \\
\text { TRP }\end{array}$ & $\mathrm{R}^{*}, \AA$ \\
\hline \multicolumn{6}{|c|}{ atoms of polar groups } \\
\hline OG Ser 14 & NE1 & 6.86 & Ser 14 & NE1 & 6.61 \\
\hline NE Arg 18 & $\mathrm{O}$ & 4.16 & Arg 18 & $\mathrm{C}$ & 6.17 \\
\hline NH1 Arg 18 & $\mathrm{O}$ & 2.95 & Arg 18 & $\mathrm{C}$ & 6.46 \\
\hline NH2 Arg 18 & $\mathrm{O}$ & 5.18 & OG1 Thr 19 & $\mathrm{O}$ & 6.16 \\
\hline OE1 Glu 42 & $\mathrm{~N}$ & 5.44 & NH1 Arg 41 & $\mathrm{O}$ & 6.26 \\
\hline OE2 Glu 42 & $\mathrm{~N}$ & 6.15 & OG Ser 95 & $\mathrm{CH} 2$ & 6.02 \\
\hline OG Ser 95 & $\mathrm{CH} 2$ & 6.67 & OG1 Thr 97 & $\mathrm{CH} 2$ & 6.78 \\
\hline ND1 His 98 & CZ3 & 6.87 & ND1 His 98 & CZ3 & 6.75 \\
\hline NZ Lys 121 & NE1 & 5.16 & NZ Lys 121 & NE1 & 4.83 \\
\hline \multirow[t]{5}{*}{ O HOH 318A } & CZ3 & 6.87 & O HOH 1006 & $\mathrm{O}$ & 4.90 \\
\hline & & & HOH 1047 & $\mathrm{C}$ & 4.33 \\
\hline & & & HOH 1066 & $\mathrm{~N}$ & 5.20 \\
\hline & & & HOH 1080 & CD1 & 6.62 \\
\hline & & & HOH 1107 & $\mathrm{O}$ & 6.46 \\
\hline \multicolumn{6}{|c|}{ atoms of peptide bonds } \\
\hline O Leu 13 & NE1 & 2.87 & O Leu 13 & NE1 & 2.74 \\
\hline O Ser 14 & NE1 & 5.05 & O Glu 12 & NE1 & 6.62 \\
\hline N Ser 14 & NE1 & 4.86 & O Ser 14 & NE1 & 5.11 \\
\hline O Gly 15 & $\mathrm{~N}$ & 3.66 & N Ser 14 & NE1 & 4.74 \\
\hline N Gly 15 & NE1 & 4.43 & O Gly 15 & $\mathrm{~N}$ & 3.08 \\
\hline N Pro 16 & $\mathrm{~N}$ & 3.54 & N Gly 15 & CD1 & 4.19 \\
\hline O Pro 16 & $\mathrm{~N}$ & 2.25 & N Pro 16 & $\mathrm{~N}$ & 3.15 \\
\hline N Arg 18 & $\mathrm{C}$ & 1.32 & O Pro 16 & $\mathrm{~N}$ & 2.21 \\
\hline O Arg 18 & $\mathrm{C}$ & 3.95 & N Arg 18 & $\mathrm{C}$ & 1.33 \\
\hline O Phe 40 & $\mathrm{O}$ & 3.26 & O Arg 18 & $\mathrm{C}$ & 3.93 \\
\hline N Phe 40 & $\mathrm{O}$ & 5.10 & N Thr 19 & $\mathrm{C}$ & 4.49 \\
\hline N Glu 42 & $\mathrm{O}$ & 4.22 & O Thr 19 & $\mathrm{C}$ & 6.91 \\
\hline O Glu 42 & $\mathrm{CD} 1$ & 5.73 & O Tyr 39 & $\mathrm{O}$ & 6.37 \\
\hline N Leu 43 & CD1 & 4.02 & O Phe 40 & $\mathrm{O}$ & 3.24 \\
\hline O Leu 43 & CD1 & 4.49 & N Phe 40 & $\mathrm{O}$ & 5.11 \\
\hline O Ser 95 & $\mathrm{CH} 2$ & 4.14 & N Arg 41 & $\mathrm{O}$ & 3.64 \\
\hline N Ser 95 & $\mathrm{CH} 2$ & 5.77 & O Arg 41 & $\mathrm{O}$ & 3.53 \\
\hline N Arg 96 & $\mathrm{CH} 2$ & 4.71 & N Glu 42 & $\mathrm{O}$ & 4.07 \\
\hline O Arg 96 & $\mathrm{CH} 2$ & 6.11 & O Glu 42 & $\mathrm{CB}$ & 5.54 \\
\hline N Thr 97 & $\mathrm{CH} 2$ & 5.09 & N Leu 43 & CD1 & 3.86 \\
\hline O Thr 97 & CZ3 & 3.76 & O Leu 43 & CD1 & 4.44 \\
\hline O His 98 & $\mathrm{CH} 2$ & 4.10 & N Val 44 & CD1 & 6.03 \\
\hline N His 98 & CZ3 & 4.14 & O Tyr 55 & $\mathrm{O}$ & 5.97 \\
\hline N Leu 99 & CZ3 & 3.74 & O Ser 95 & $\mathrm{CH} 2$ & 3.55 \\
\hline O Leu 99 & CZ3 & 5.57 & N Ser 95 & $\mathrm{CH} 2$ & 5.32 \\
\hline O Phe 119 & CZ3 & 3.85 & N Arg 96 & $\mathrm{CH} 2$ & 5.48 \\
\hline N Phe 119 & CE3 & 5.75 & O Arg 96 & CZ2 & 4.43 \\
\hline O Val 120 & CA & 3.38 & N Thr 97 & $\mathrm{CH} 2$ & 4.59 \\
\hline N Val 120 & CE3 & 3.92 & O Thr 97 & CZ3 & 4.10 \\
\hline O Lys 121 & CA & 6.07 & O His 98 & $\mathrm{CH} 2$ & 3.67 \\
\hline N Lys 121 & CE3 & 3.51 & $\mathrm{~N}$ His 98 & $\mathrm{CH} 2$ & 3.82 \\
\hline
\end{tabular}


N Leu 122

O Leu 122
CA

$\mathrm{O}$
4.61
5.97

5.97
N Leu 99

O Leu 99

N Val 100

O Phe 119

N Phe 119

O Val 120

N Val 120

O Lys 121

N Lys 121

N Gly $121+$

$\begin{array}{ll}\text { CZ3 } & 3.64 \\ \text { CZ3 } & 5.59 \\ \text { CZ3 } & 6.72 \\ \text { CZ3 } & 3.53 \\ \text { CE3 } & 5.67 \\ \text { CA } & 3.51 \\ \text { CE3 } & 3.73 \\ \text { CD2 } & 6.58 \\ \text { CE3 } & 3.77 \\ \text { CZ3 } & 6.22\end{array}$

atoms of nonpolar groups and aromatic residues

CB Pro 16

CB Phe 40

CE1 Phe 45

CB His 98

CB Phe 119

CD2 Leu 13

CB Leu 43

CD1 Leu 94

CB Leu 99

CB Val 120

CB Lys 121

CD1 Leu 122
$\mathrm{N}$

$\mathrm{O}$

NE1

CZ3

CE3

CZ2

CD1

$\mathrm{CH} 2$

CZ3

CE3

CD2/CE2

C
3.49

3.74

4.61

5.32

4.13

4.17

3.65

4.26

3.80

5.51

3.83

4.69

CB Pro 16
CB Phe 40
CE1 Phe 45
CB His 98
CB Phe 119
CB Leu 13
CB Leu 43
CD1 Leu 94
CB Leu 99
CB Val 120
CD Lys 121

CB Pro 16

CE1 Phe 45

CB His 98

CB Phe 119

CB Leu 13

CB Leu 43

CD1 Leu 94

CB Val 120

CD Lys 121
3.73

3.72

4.06

5.18

4.08

4.65

3.52

4.37

3.75

5.26

3.61 3

* $\mathrm{R}$ is the minimal distance between a atom of residue of the microenvironment of tryptophan residue and the nearest atom of its indole ring. 


\section{Table 4(on next page)}

Table 4 . Characteristics of the Trp 64 microenvironment in bOBPwt 
1 Table 4. Characteristics of the Trp 64 microenvironment in bOBPwt.

2

\begin{tabular}{ccc}
\hline $\begin{array}{c}\text { atom of the } \\
\text { microenvironment }\end{array}$ & atom of TRP & $\mathrm{R}^{*}, \AA$ \\
\hline
\end{tabular}

$$
\text { atoms of polar groups }
$$

OH Tyr 39

OG Ser 57

$\mathrm{CH} 2 \quad 5.35$

NZ Lys 59

CE3 $\quad 5.05$

NZ Lys 63

NE1 $\quad 4.55$

NZ Lys 65

$\mathrm{N} \quad 6.58$

ND1 His 157

$\mathrm{O}$

5.23

NE2 His 157

CD1

3.27

OE1 Glu 159

CD1

4.22

OE2 Glu 159

$\mathrm{CH} 2$

5.16

$\mathrm{HOH} 205 \mathrm{~A}$

$\mathrm{CH} 2$

4.56

CH2 $\quad 4.04$

$\mathrm{HOH} 231 \mathrm{~A}$

CZ3 $\quad 5.70$

$\mathrm{HOH} 247 \mathrm{~A}$

$\mathrm{HOH} 282 \mathrm{~A}$

CH2 $\quad 5.80$

$\mathrm{HOH} 289 \mathrm{~A}$

CD2 $\quad 3.74$

$\mathrm{HOH} 298 \mathrm{~A}$

HOH 328A

$\begin{array}{ll}\mathrm{CB} & 4.18 \\ \mathrm{C} & 3.74\end{array}$

CZ3 3.56

NE1 2.88

atoms of peptide bonds

N Tyr 39

O Tyr 39

N Ser 57

O Ser 57

$\mathrm{N}$ Val 58

O Val 58

N Lys 59

O Lys 59

$\mathrm{N} \operatorname{Arg} 60$

O Arg 60

N Lys 63

O Lys 63

N Lys 65

O Lys 65

O Pro 156

CZ3

CZ3

CZ3

6.78

$\mathrm{CE} 3 / \mathrm{CZ3} \quad 4.00$

$\begin{array}{ll}\mathrm{C} & 3.67\end{array}$

$\mathrm{CE} 3 / \mathrm{CZ3} \quad 3.78$

CA $\quad 6.52$

$\begin{array}{ll}\mathrm{N} & 6.11 \\ \mathrm{~N} & \end{array}$

$\mathrm{N} \quad 5.63$

$\mathrm{N} \quad 3.61$

$\begin{array}{ll}\mathrm{N} & 2.25 \\ \mathrm{C}\end{array}$

$\begin{array}{ll}\text { C } & 1.32\end{array}$

$\begin{array}{ll}\mathrm{C} & 4.07\end{array}$

CE2 6.63

$\mathrm{N}$ His 157

NE1 5.98

$\begin{array}{lll}\text { O His } 157 & \text { CZ2 } & 5.90\end{array}$

$\begin{array}{lll}\text { N Pro } 158 & \text { CZ2 } & 4.47\end{array}$

$\begin{array}{lll}\text { O Pro } 158 & \text { CZ2 } & 6.07\end{array}$

$\begin{array}{lll}\text { N Glu } 159 & \text { CZ2 } & 4.12\end{array}$

O Glu $159 \quad$ NE1 3.31

atoms of nonpolar groups and aromatic residues

CE1 Tyr 39

CE1 His 155

$\mathrm{CH} 2 \quad 3.88$

CE1 His 157

CE3 $\quad 6.40$

CD Pro 158

CD2 3.52

CB Val 58

CB Lys 63

$\mathrm{CZ2} \quad 3.69$

$\mathrm{CZ3} \quad 5.50$

$\mathrm{N} \quad 3.23$



CB Lys 65
$\mathrm{C}$
3.46

$3 * \mathrm{R}$ is the minimal distance between a residue involved in the microenvironment of tryptophan

4 residue and its indole ring.

5 


\section{Table 5(on next page)}

Table 5 . Characteristics of the Trp 133 microenvironment in bOBPwt andGCC-bOBP 
1 Table 5. Characteristics of the Trp 133 microenvironment in bOBPwt and GCC-bOBP.

\begin{tabular}{|c|c|c|c|c|c|}
\hline \multicolumn{3}{|c|}{ bOBPwt } & \multicolumn{3}{|c|}{ GCC-bOBP } \\
\hline $\begin{array}{l}\text { atoms of the } \\
\text { microenvironment }\end{array}$ & $\begin{array}{c}\text { atom of } \\
\text { TRP }\end{array}$ & $\begin{array}{c}\mathrm{R}^{*} \\
\AA\end{array}$ & $\begin{array}{l}\text { atoms of the } \\
\text { microenvironment }\end{array}$ & $\begin{array}{c}\text { atom } \\
\text { of } \\
\text { TRP }\end{array}$ & $\mathrm{R}^{*}, \AA$ \\
\hline \multicolumn{6}{|c|}{ atoms of polar groups } \\
\hline OH Tyr 21B & $\mathrm{CZ2}$ & 4.36 & OH Tyr 21 & NE1 & 5.03 \\
\hline OG1 Thr 136 & $\mathrm{CA}$ & 4.35 & OG1 Thr 136 & $\mathrm{CA}$ & 4.44 \\
\hline NZ Lys 143 & CE3 & 3.35 & HOH 1087 & $\mathrm{CH} 2$ & 4.99 \\
\hline $\mathrm{HOH} 218 \mathrm{~A}$ & $\mathrm{CE} 3 / \mathrm{CZ} 3$ & 4.69 & & & \\
\hline $\mathrm{HOH} 254 \mathrm{~A}$ & NE1 & 3.94 & & & \\
\hline $\mathrm{HOH} 232 \mathrm{~B}$ & NE1 & 3.27 & & & \\
\hline $\mathrm{HOH} 283 \mathrm{~B}$ & $\mathrm{CZ2}$ & 5.83 & & & \\
\hline \multicolumn{6}{|c|}{ atoms of peptide bonds } \\
\hline N Leu 129 & $\mathrm{~N}$ & 6.23 & O Leu 129 & $\mathrm{~N}$ & 2.97 \\
\hline O Leu 129 & $\mathrm{~N}$ & 3.10 & O Phe 132 & $\mathrm{~N}$ & 2.26 \\
\hline N Glu 130 & $\mathrm{CD} 1$ & 4.75 & N Lys 134 & $\mathrm{C}$ & 1.33 \\
\hline O Glu 130 & $\mathrm{~N}$ & 3.33 & & & \\
\hline N Phe 132 & $\mathrm{~N}$ & 2.85 & & & \\
\hline O Phe 132 & $\mathrm{~N}$ & 2.26 & & & \\
\hline N Lys 134 & $\mathrm{C}$ & 1.33 & & & \\
\hline O Lys 134 & $\mathrm{O}$ & 3.28 & & & \\
\hline N Thr 136 & $\mathrm{O}$ & 3.37 & & & \\
\hline O Thr 136 & $\mathrm{O}$ & 5.01 & & & \\
\hline N Lys 143 & $\mathrm{CZ3}$ & 5.56 & & & \\
\hline O Lys 143 & $\mathrm{CH} 2$ & 5.36 & & & \\
\hline N Val 146 & $\mathrm{CH} 2$ & 6.50 & & & \\
\hline O Val 146 & $\mathrm{CH} 2$ & 6.63 & & & \\
\hline \multicolumn{6}{|c|}{ atoms of nonpolar groups and aromatic residues } \\
\hline CE2 Tyr 21B & $\mathrm{CZ2}$ & 4.17 & CE2 Tyr 21 & NE1 & 3.96 \\
\hline C Phe 132 & $\mathrm{~N}$ & 1.34 & C Phe 132 & $\mathrm{~N}$ & 1.33 \\
\hline C Leu 129 & $\mathrm{CD} 1$ & 3.90 & C Leu 129 & CD1 & 4.15 \\
\hline CA Lys 134 & $\mathrm{C}$ & 2.43 & CG2Val 146 & $\mathrm{CZ3}$ & 3.98 \\
\hline CG2 Val 146 & $\mathrm{CZ2}$ & 3.69 & CA Lys 143 & $\mathrm{CZ3}$ & 4.55 \\
\hline CG Lys 143 & $\mathrm{CZ3}$ & 3.75 & & & \\
\hline
\end{tabular}

* $\mathrm{R}$ is the minimal distance between a residue involved in the microenvironment of tryptophan residue and its indole ring. 


\section{Table 6(on next page)}

Table 6 . The evaluation of secondary structure of the recombinant bOBPwt andits mutant forms using Provencher's algorithm (Provencher\& Glockner 1981) 
1 Table 6. The evaluation of secondary structure of the recombinant bOBPwt and its mutant forms

2 using Provencher's algorithm (Provencher \& Glockner 1981).

\begin{tabular}{lcccr} 
& $\alpha$ & $\beta$ & turn & unordered \\
bOBPwt & 0.133 & 0.359 & 0.204 & 0.297 \\
bOBPwt/OCT & 0.113 & 0.354 & 0.207 & 0.311 \\
bOBP-Gly121+ & 0.085 & 0.400 & 0.208 & 0.303 \\
bOBP-Gly121/OCT & 0.112 & 0.407 & 0.200 & 0.272 \\
GCC-bOBP & 0.134 & 0.353 & 0.208 & 0.293 \\
GCC-bOBP/OCT & 0.145 & 0.344 & 0.217 & 0.288 \\
GCC-bOBP W17F & 0.077 & 0.415 & 0.209 & 0.299 \\
GCC-bOBP W17F/OCT & 0.087 & 0.429 & 0.206 & 0.276 \\
GCC-bOBP W133F & 0.154 & 0.356 & 0.206 & 0.269 \\
\hline GCC-bOBP W133F/OCT & 0.172 & 0.352 & 0.200 & 0.275
\end{tabular}

3 


\section{1}

3D structure of bOBP

Figure 1. 3D structure of bOBP. The individual subunits in the protein are in gray and orange. The tryptophan residues in the different subunits are indicated in red and blue. The Lys 121 residue after which an extra glycine residue are inserted in the mutant form bOBPGly121+ is drown in green. Additionally the residues Trp 64 and His 156 (yellow) are substituted for cysteine in the mutant form GCC-bOBP. The drawing was generated based on the 1OBP file (Tegoni et al. 1996) from PDB (Dutta et al. 2009) using the graphic software VMD (Hsin et al. 2008) and Raster3D (Merritt \& Bacon 1977) .

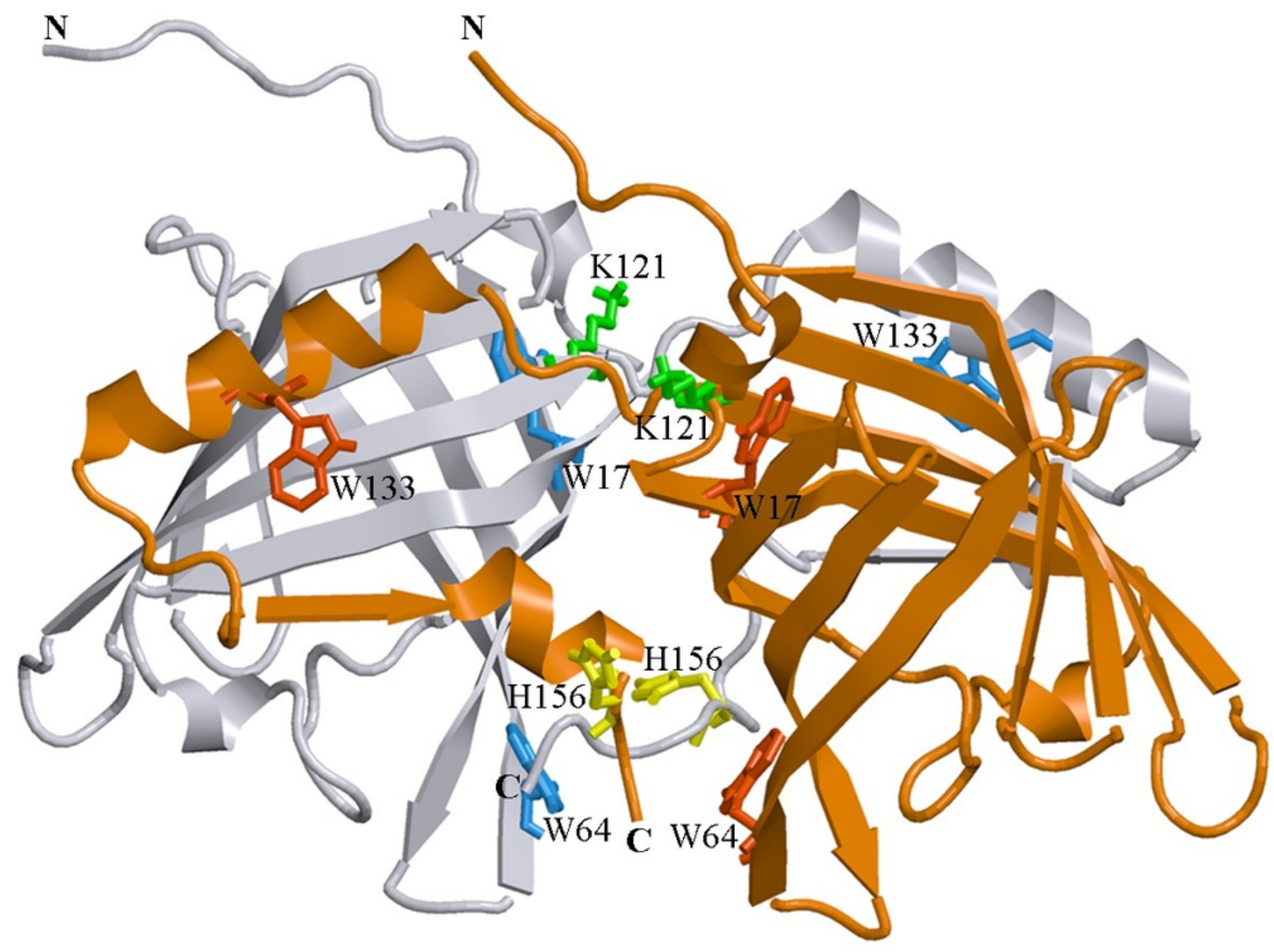


Figure 2 (on next page)

Sequence peculiarities of various bPDB forms.

Figure 2. Sequence peculiarities of various bPDB forms. The comparison of the primary sequence for the recombinant bOBPwt and its mutant forms bOBP-Gly $121+$ and GCC- bOBP, which are not able to form domain-swapped dimers. The mutant forms GCCbOBP-W17F and GCC-bOBP-W133F designed to contain a single tryptophan residue were produced to investigate the peculiarities of the microenvironment of the tryptophan residues. 
1.Gob tryptophan residues.

$\begin{array}{lrrrrrr}1 & 10 & 20 & 30 & 40 & 50 & 60 \\ \text { AQEEEAEQNL } & \text { SELSGPWRTV } & \text { YIGSTNPEKI } & \text { QENGPFRTYF } & \text { RELVFDDEKG } & \text { TVDFYFSVKR } \\ 61 & 70 & 80 & 90 & 100 & 110 & 120 \\ \text { DGKWKNVHVK } & \text { ATKQDDGTYV } & \text { ADYEGQNVFK } & \text { IVSLSRTHLV } & \text { AHNINVDKHG } & \text { QTTELTGLFV } \\ 121 & 130 & 140 & 150 & 159 & \end{array}$

2. bOBP-Gly121+ - the introduction of an extra glycine residue after the bOBP residue 121 is proposed to inhibit dimer formation as a result of the increased mobility of the loop connecting $\alpha$-helix and $8^{\text {th }} \beta$-strand of the $\beta$-barrel. The protein contains 3 tryptophan residues as well.

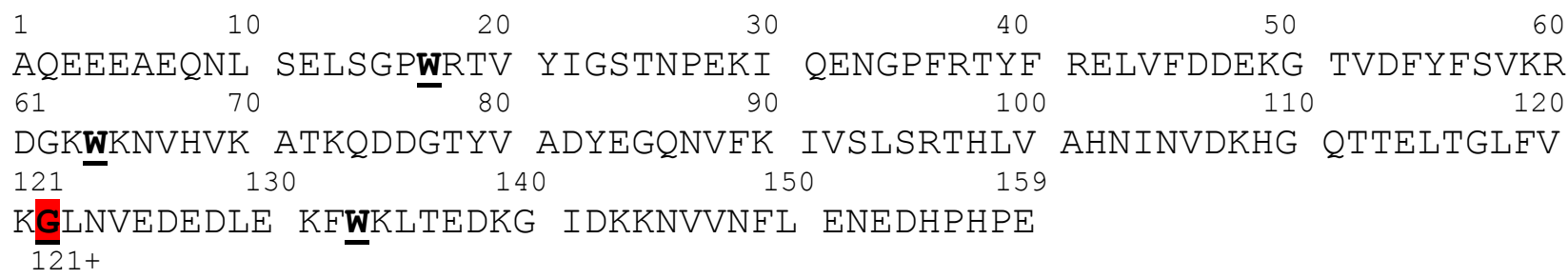

3. GCC- bOBP (bOBP-Gly121+-W64C-H155C) - the substitutions W64C and H155C result in the restoration of the disulfide bond which is necessary for the additional stabilization of the protein. The protein has only 2 tryptophan residues.

$\begin{array}{lllll}1 & 10 & 20 & 30 & 40\end{array}$

AQEEEAEQNL SELSGPWRTV YIGSTNPEKI QENGPFRTYF RELVFDDEKG TVDFYFSVKR

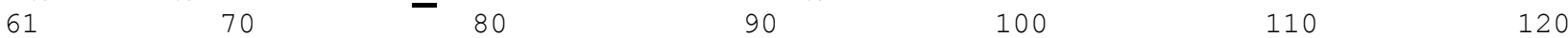

DGKCKNVHVK ATKQDDGTYV ADYEGQNVFK IVSLSRTHLV AHNINVDKHG QTTELTGLFV

$121 \quad 130 \quad 140 \quad 150 \quad 159$

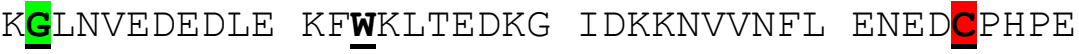
$121+$

4. GCC-bOBP-W17F (bOBP-Gly121+-W64C-H155C-W17F) - the protein contains a single tryptophan residue which allows the investigation of the features of the microenvironment of this residue.

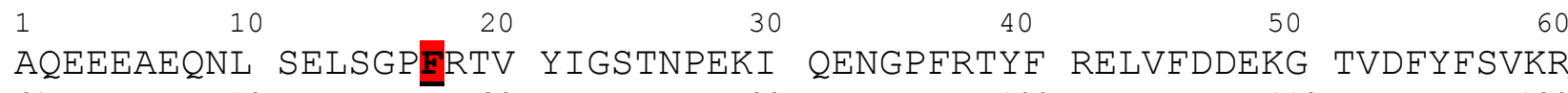

$\begin{array}{lrrrrr}61 & 70 & 80 & 90 & 100 & 110\end{array}$
DGKCGKNVHVK ATKQDDGTYV ADYEGQNVFK IVSLSRTHLV AHNINVDKHG QTTELTGLFV $121 \quad 130 \quad 140 \quad 150 \quad 159$

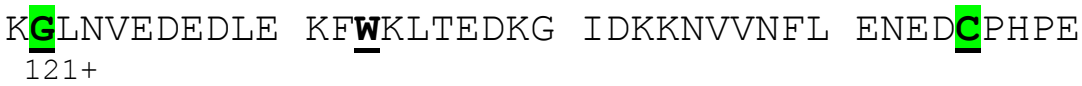

5 GCC-bOBP-W133F (bOBP/Gly121+/W64C/H155C/W133F) - the protein has a single tryptophan residue as well.

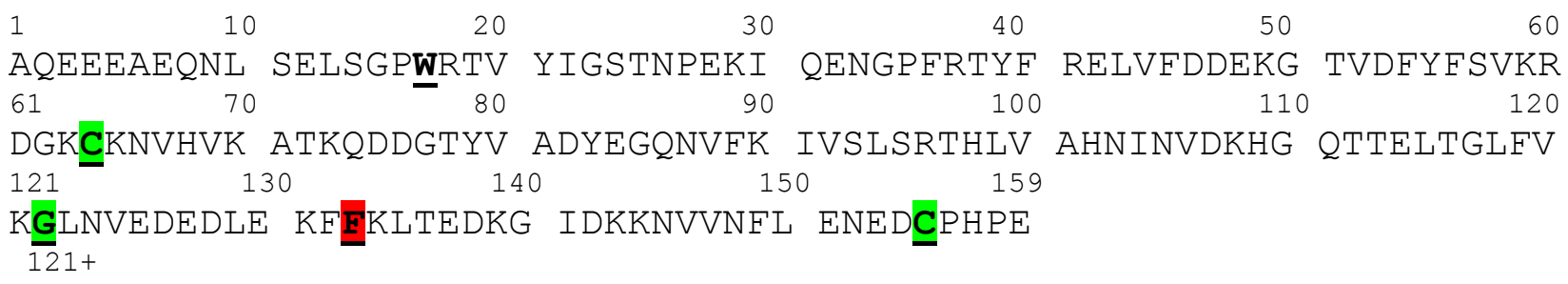


3

Intrinsic disorder propensity of the wild type bOBP and its mutants .

Figure 3. Intrinsic disorder propensity of the wild type bOBP and its mutants . A. Per-residue disorder propensity of the wild type bOBP evaluated by members of the PONDR family, PONDR ${ }^{\circledR}$ VLXT (Stepanenko et al. 2015) (green line), PONDR ${ }^{\circledR}$ VSL2 (Stepanenko et al. 2014a) (blue line), PONDR ${ }^{\circledR}$ FIT (Xue et al. 2010) (red line) and PONDR ${ }^{\circledR}$ VL3 (pink line). Localization of known elements of the bOBP secondary structure is shown by colored bars at the bottom of the plot. Light pink shadow around the PONDR ${ }^{\circledR}$ FIT curve represents distribution of errors in the disorder score evaluation. B . Effects of mutations on the intrinsic disorder propensity of bOBP evaluated by PONDR ${ }^{\circledR}$ VSL2. 

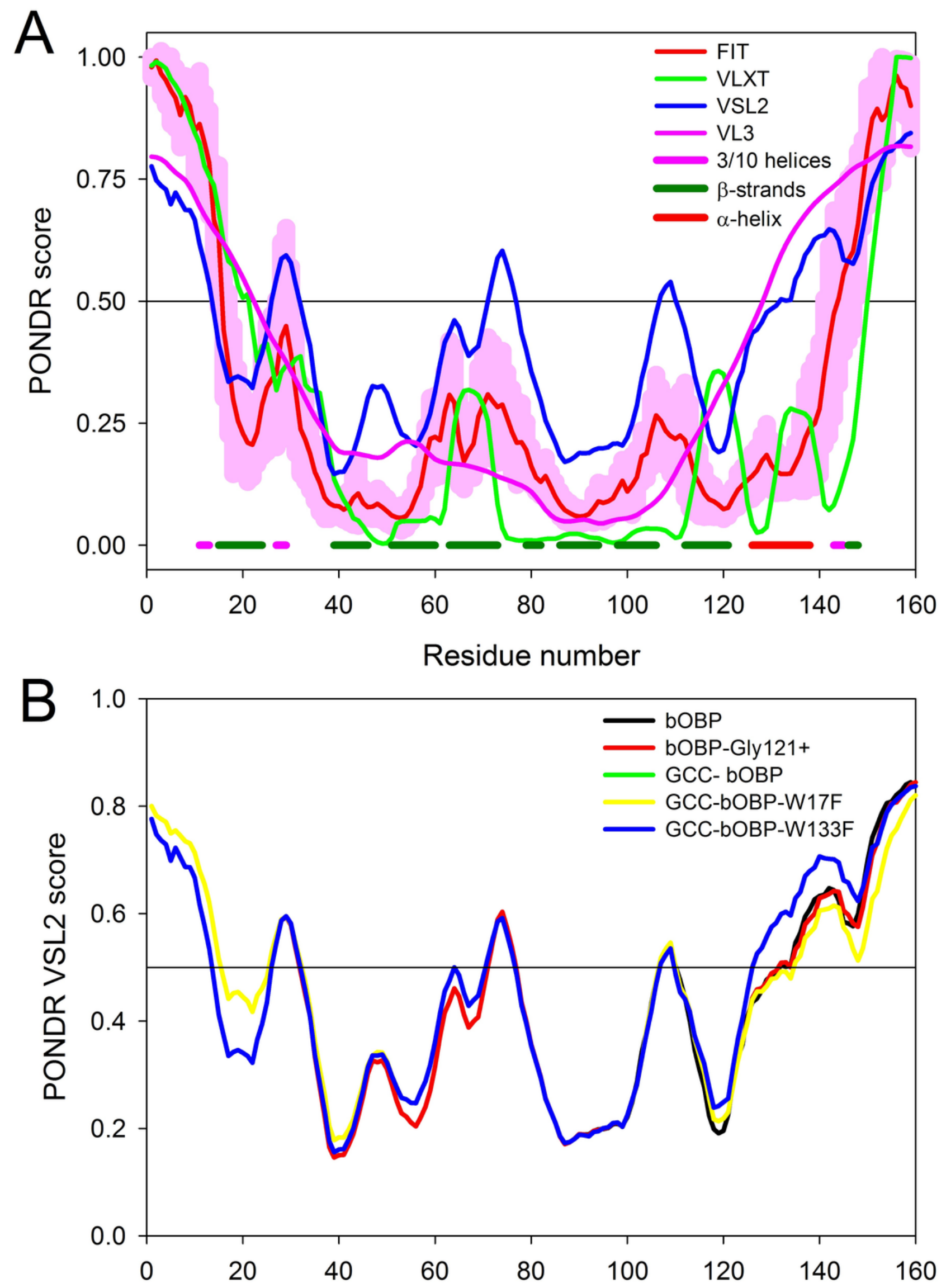


\section{Figure 4 (on next page)}

$\mathrm{GdnHCl-induced} \mathrm{conformational} \mathrm{changes} \mathrm{in} \mathrm{bOBP} \mathrm{(data} \mathrm{for} \mathrm{this} \mathrm{figure} \mathrm{aretaken} \mathrm{from}$ (Stepanenko et al. 2014b)).

Figure 4. Hydrodynamic characteristics of the bOBP and its mutants. The changes of hydrodynamic dimensions of recombinant bOBP (1) and its mutant forms bOBP-Gly121+ (2), GCC-bOBP (3), GCC-bOBP-W17F (4) and GCC-bOBP-W133F (5) in the absence (solid lines) and the presence of OCT (dotted lines). 


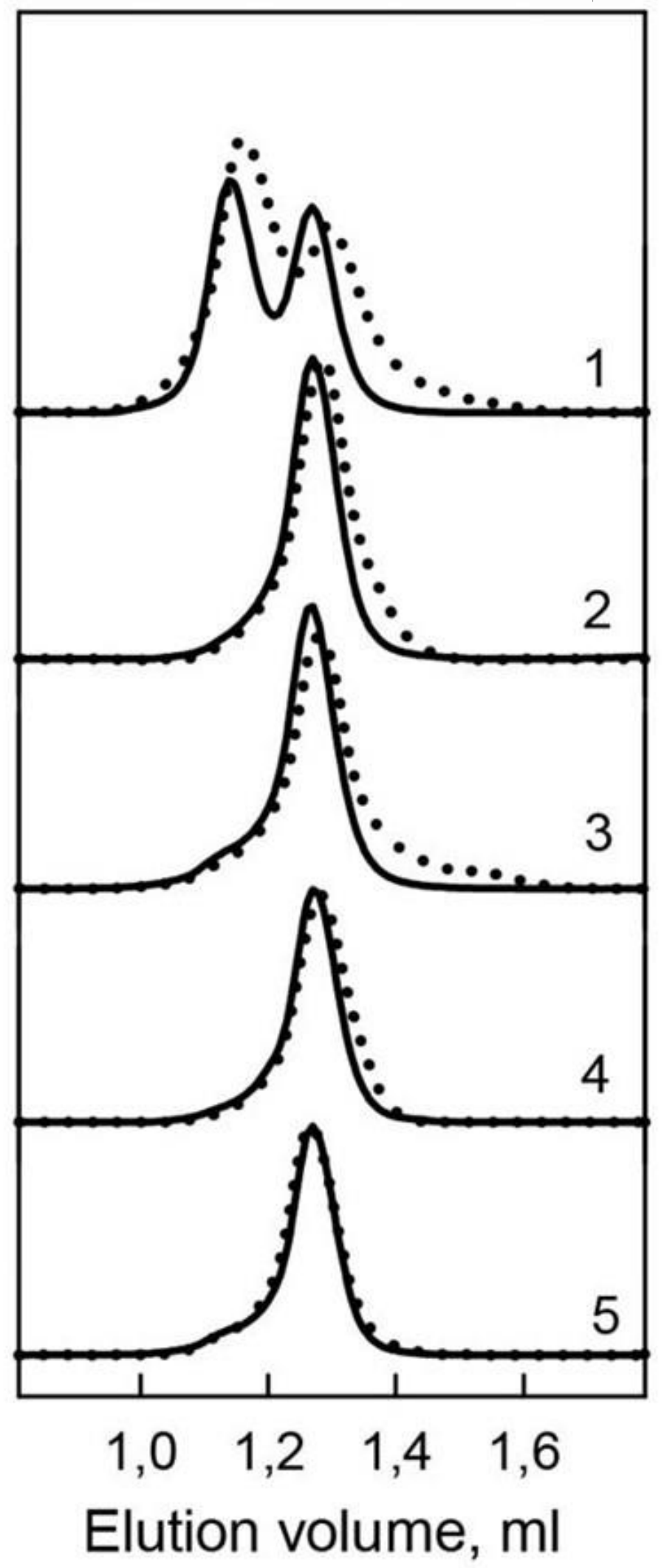


5

Tertiary structure changes for bOBP (red) and its mutant formsbOBP-Gly $121+$ (green), GCC-bOBP (blue), GCC-bOBP-W17F (gray) and GCC-bOBP-W133F(dark yellow) in different structural states

Figure 5. Tertiary structure changes for bOBP (red) and its mutant forms bOBP-Gly121+ (green), GCC-bOBP (blue), GCC-bOBP-W17F (gray) and GCC-bOBP-W133F (dark yellow) in different structural states are indicated by intrinsic tryptophan fluorescence $\left(\lambda_{\mathrm{ex}}=297 \mathrm{~nm}\right)$. The spectra shown are for the protein in buffered solution (solid line), in the presence of natural ligand OCT (dotted line) and in the presence of $3.5 \mathrm{M} \mathrm{GdnHCl}$ (dashed line). The corresponding spectra in light blue were obtained as a sum of the spectra for GCC-bOBP-W17F and GCC-bOBP-W133F.

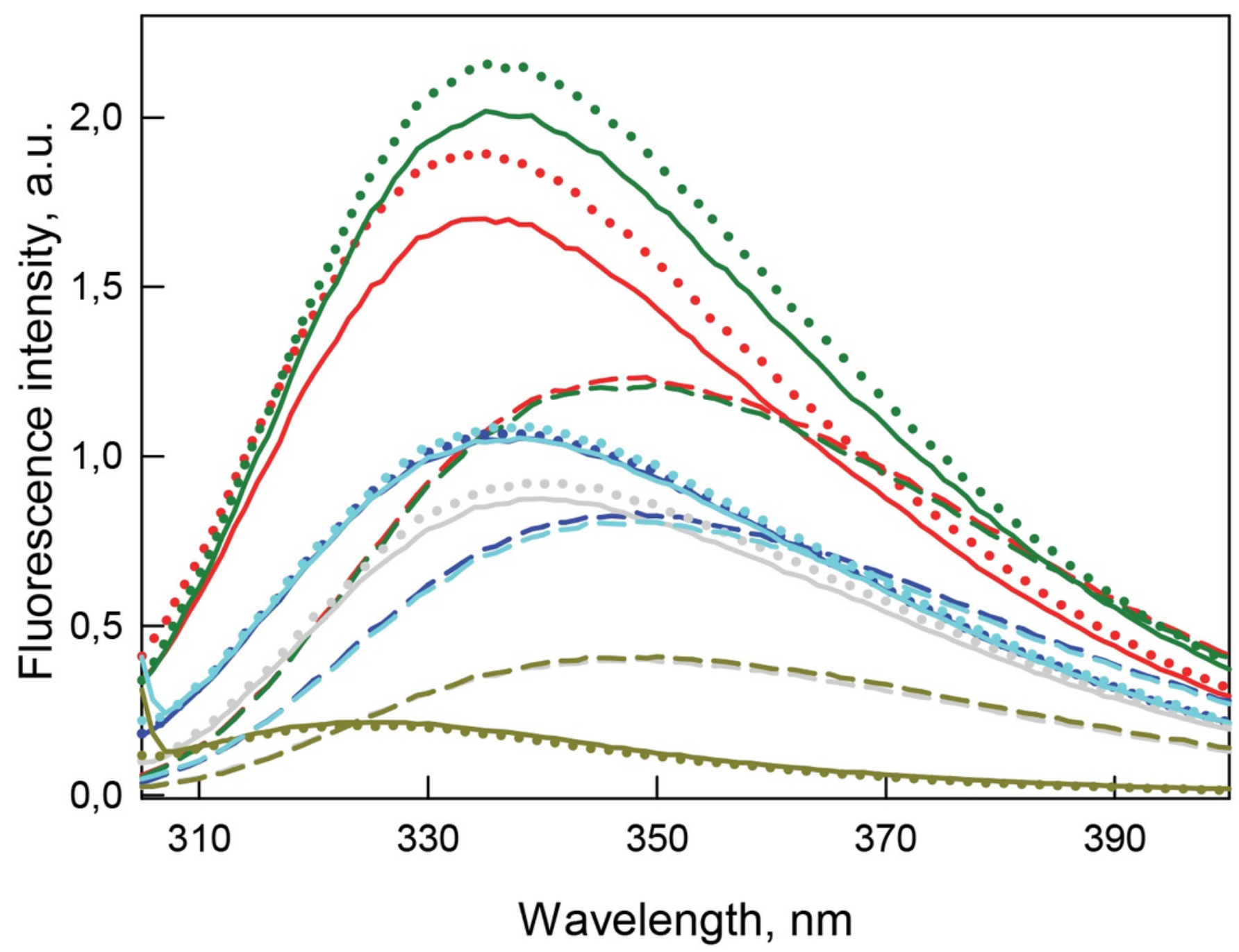


6

Tertiary structure for bOBP (red) and its mutant forms bOBP-Gly $121+$ (green), GCC-bOBP (blue), GCC-bOBP-W17F (gray) and GCC-bOBP-W133F (dark yellow)

Figure 6. Tertiary structure for bOBP (red) and its mutant forms bOBP-Gly121+ (green), GCCbOBP (blue), GCC-bOBP-W17F (gray) and GCC-bOBP-W133F (dark yellow) in buffered solution are indicated by near-UV CD spectra. The spectrum in light blue was obtained as a sum of the spectra for GCCbOBP-W17F and GCC-bOBP-W133F.

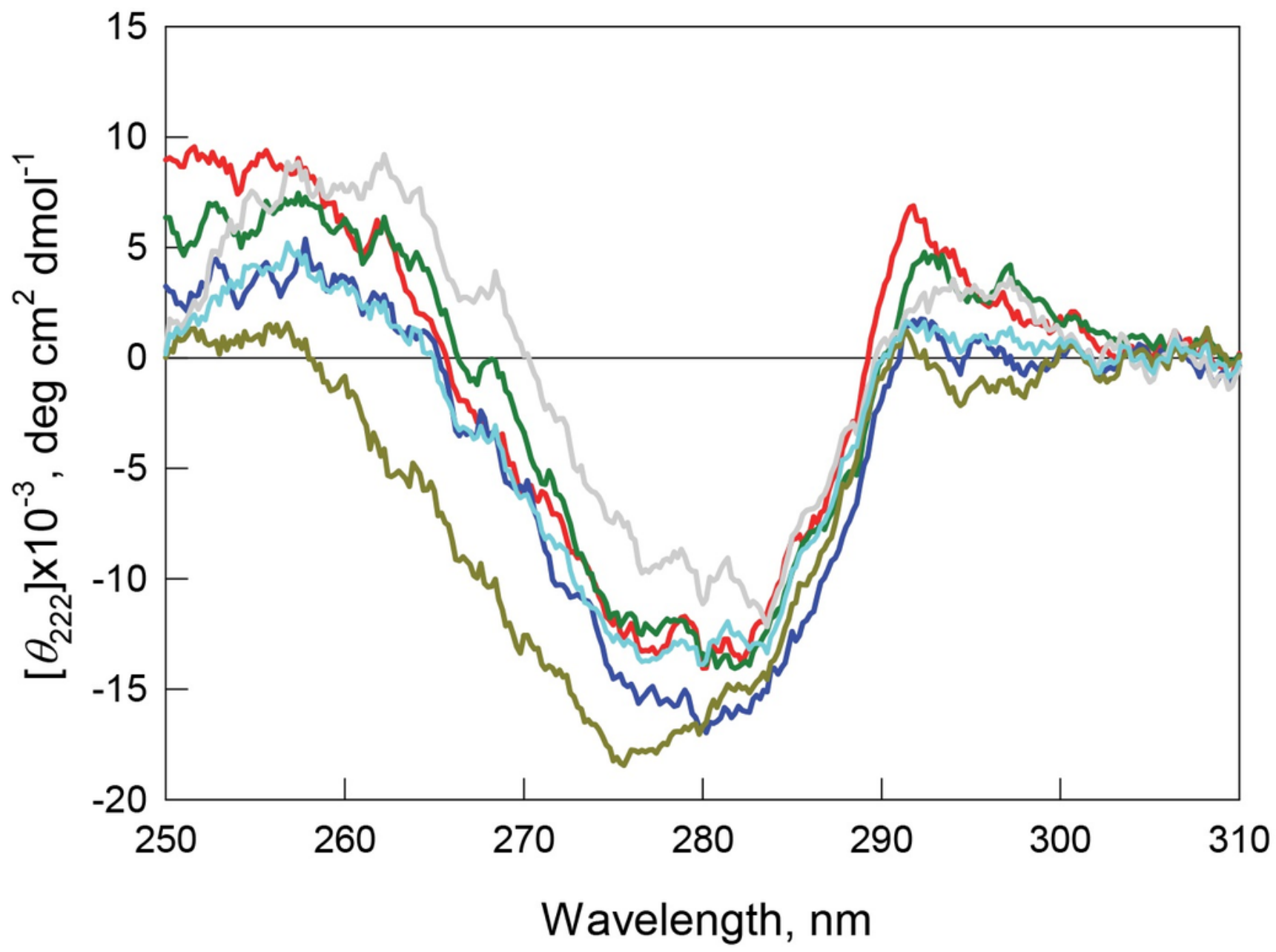




\section{7}

The microenvironment of Trp $17(A)$ and Trp $64(B)$ in bOBP.

Figure 7. The microenvironment of Trp $17(A)$ and Trp $64(B)$ in bOBP. The spatial orientation of lysine residues relative the indole ring of tryptophan residues is shown. The drawing was generated based on the 1OBP file (Tegoni et al. 1996) from PDB (Dutta et al. 2009) using the graphic software VMD (Hsin et al. 2008) and Raster3D (Merritt \& Bacon 1977) .

$\boldsymbol{A}$

$B$
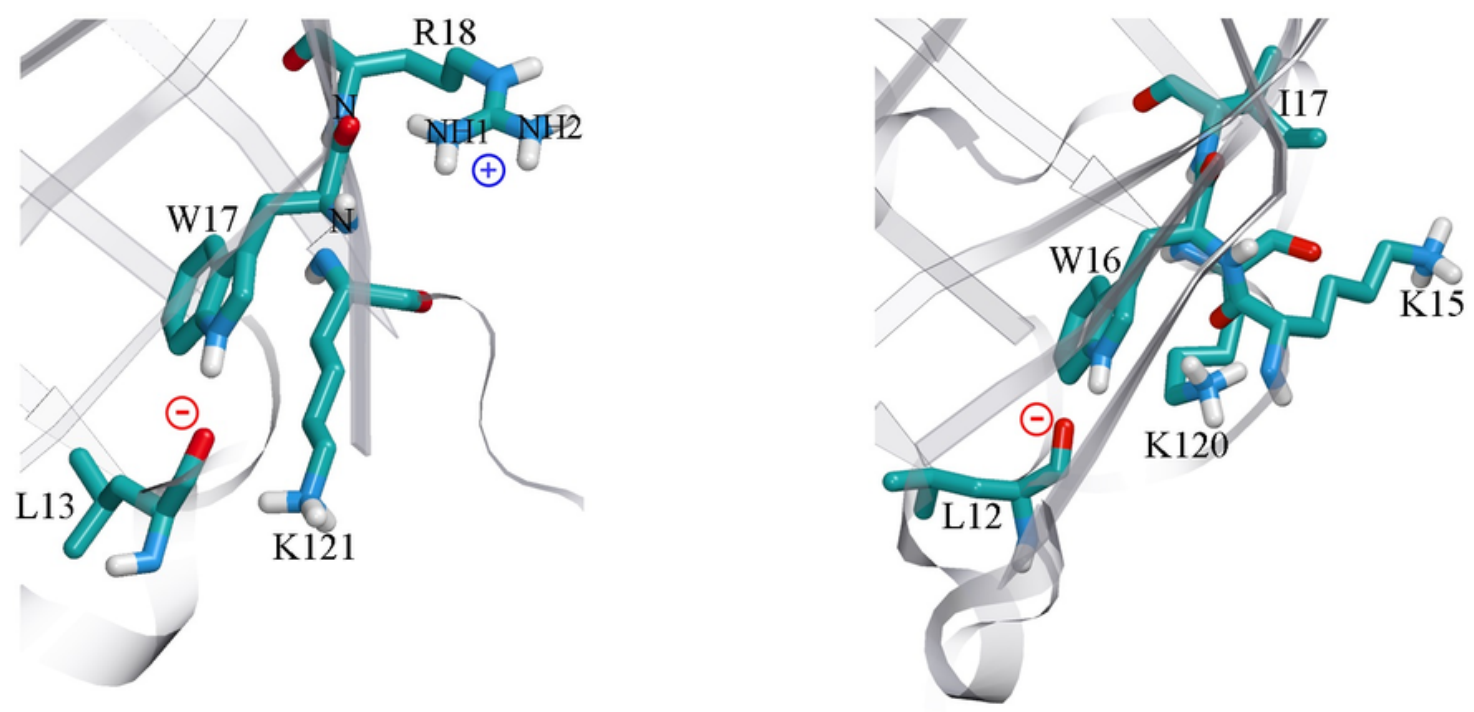
8

Secondary structure for bOBP (red) and itsmutant forms bOBP-Gly121+ (green), GCC-bOBP (blue), GCCbOBP-W17F (gray) andGCC-bOBP-W133F (dark yellow)

Figure 8. Secondary structure for bOBP (red) and its mutant forms bOBP-Gly121+ (green), GCCbOBP (blue), GCC-bOBP-W17F (gray) and GCC-bOBP-W133F (dark yellow) in buffered solution are indicated by far-UV CD spectra.

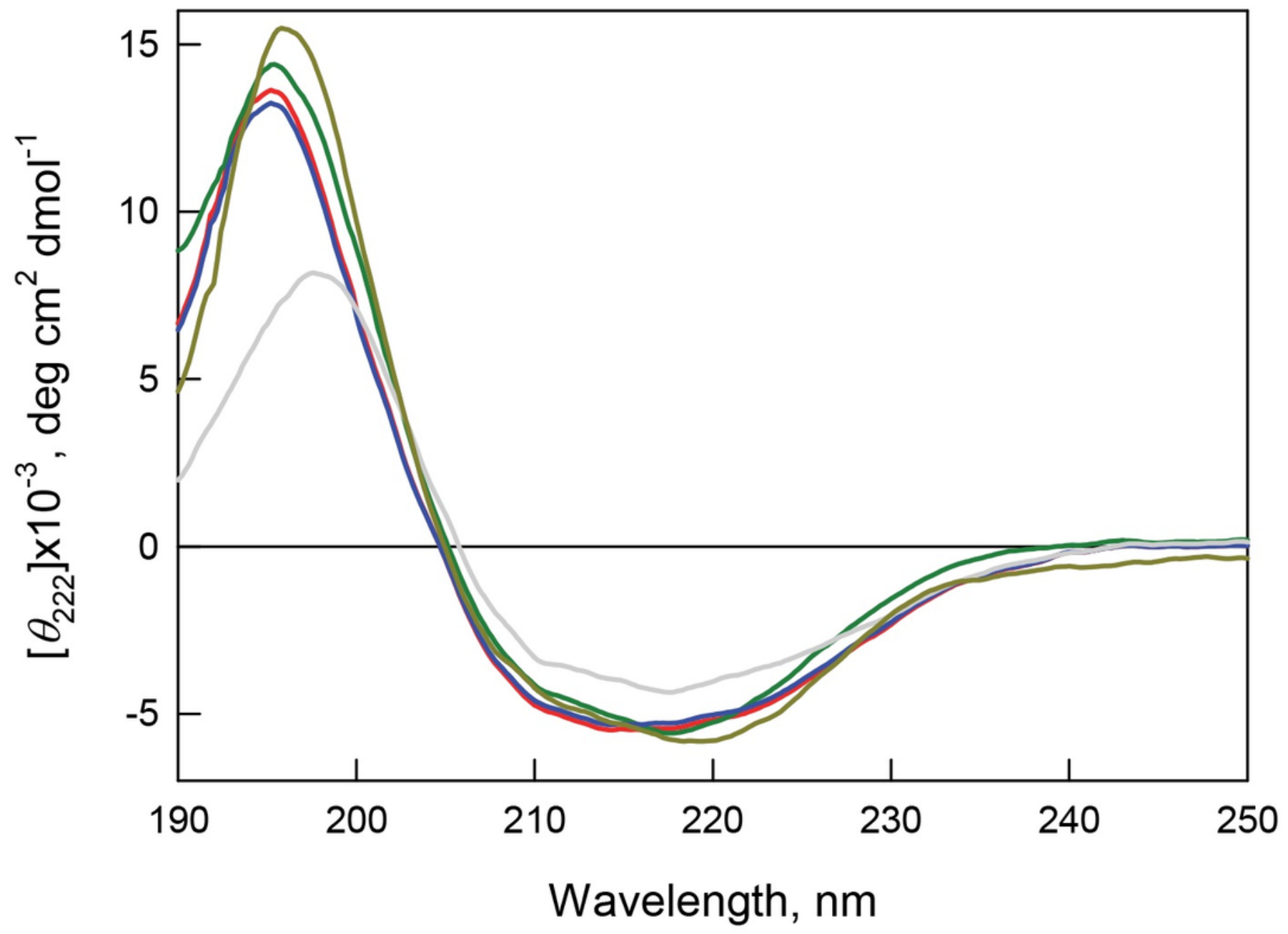

